\title{
The Role of External Factors in the Variability of the Structure of the Zooplankton Community of Small Lakes (South-East Kazakhstan)
}

\author{
Moldir Aubakirova ${ }^{1,2, *}$, Elena Krupa ${ }^{3}\left(\mathbb{D}\right.$, Zhanara Mazhibayeva ${ }^{2}$, Kuanysh Isbekov $^{2}$ and Saule Assylbekova ${ }^{2}$ \\ 1 Faculty of Biology and Biotechnology, Al-Farabi Kazakh National University, Almaty 050040, Kazakhstan \\ 2 Fisheries Research and Production Center, Almaty 050016, Kazakhstan; mazhibayeva@fishrpc.kz (Z.M.); \\ isbekov@fishrpc.kz (K.I.); assylbekova@fishrpc.kz (S.A.) \\ 3 Institute of Zoology, Almaty 050060, Kazakhstan; elena_krupa@mail.ru \\ * Correspondence: judo_moldir@mail.ru; Tel.: +7-27-3831715
}

check for updates

Citation: Aubakirova, M.; Krupa, E.; Mazhibayeva, Z.; Isbekov, K.; Assylbekova, S. The Role of External Factors in the Variability of the Structure of the Zooplankton Community of Small Lakes (South-East Kazakhstan). Water 2021, 13, 962. https://doi.org/10.3390/ w13070962

Academic Editor: Marina Marcella Manca

Received: 23 February 2021

Accepted: 29 March 2021

Published: 31 March 2021

Publisher's Note: MDPI stays neutral with regard to jurisdictional claims in published maps and institutional affiliations.

Copyright: (c) 2021 by the authors Licensee MDPI, Basel, Switzerland. This article is an open access article distributed under the terms and conditions of the Creative Commons Attribution (CC BY) license (https:/ / creativecommons.org/licenses/by/ $4.0 /)$.

\begin{abstract}
The variability of hydrochemical parameters, the heterogeneity of the habitat, and a low level of anthropogenic impact, create the premises for conserving the high biodiversity of aquatic communities of small water bodies. The study of small water bodies contributes to understanding aquatic organisms' adaptation to sharp fluctuations in external factors. Studies of biological communities' response to fluctuations in external factors can be used for bioindication of the ecological state of small water bodies. In this regard, the purpose of the research is to study the structure of zooplankton of small lakes in South-East Kazakhstan in connection with various physicochemical parameters to understand the role of biological variables in assessing the ecological state of aquatic ecosystems. According to hydrochemical data in summer 2019, the nutrient content was relatively high in all studied lakes. A total of 74 species were recorded in phytoplankton. The phytoplankton abundance varied significantly, from $8.5 \times 10^{7}$ to $2.71667 \times 10^{9}$ cells $/ \mathrm{m}^{3}$, with a biomass from 0.4 to $15.81 \mathrm{~g} / \mathrm{m}^{3}$. Shannon diversity index of phytoplankton in the lakes at high altitude varied from 1.33 to 2.39 and from 0.46 to 3.65 in the lakes at lower altitudes. The average weight of the cells of algae species varied from 0.2079 to $1.5076 \times 10^{-6} \mathrm{mg}$ in the lakes at lower altitudes, the average weight of the cells of algae species changed from 0.6682 to $1.2963 \times 10^{-6} \mathrm{mg}$ in the lakes at higher altitudes. Zooplankton was represented by 58 taxa. The total abundance of zooplankton varied from 0.05 to 169.00 thousand ind.$/ \mathrm{m}^{3}$ with biomass of $0.51-349.01 \mathrm{mg} / \mathrm{m}^{3}$. Shannon diversity of zooplankton in the lakes at lower altitude fluctuated from 0.42 to 2.32 and it was $0.66-1.77$ in the lakes at higher altitudes. The average individual mass of specimens in zooplankton in mountain lakes ranged from 0.021 to $0.037 \mathrm{mg}$ and varied from 0.002 to $0.007 \mathrm{mg}$ in other lakes. The main factors in the development of the structure of zooplankton communities in small lakes were temperature, TDS, the content of nitrates, phosphates, and the composition and biomass of planktonic algae. The hydrochemical and biological data of the investigated lakes indicated their organic pollution. Our results once again confirmed the applicability of structural variables of zooplankton in assessing water quality.
\end{abstract}

Keywords: species richness; species diversity; phytoplankton; statistical analysis; redundancy analysis

\section{Introduction}

Small lakes and reservoirs are the most common types of aquatic ecosystems in the world landscape [1]. These include lentic water bodies with a catchment area from $0.01 \mathrm{~km}^{2}$ to $0.10 \mathrm{~km}^{2}$ [2]. Different types of small water bodies feeding (atmospheric precipitation, underground runoff, river waters) lead to the high variability of some of their hydrochemical characteristics [3]. In a short period of time, phosphorus concentration in water can vary from $0.025 \mathrm{mg} / \mathrm{L}$ to $1.5 \mathrm{mg} / 1$ and higher [1]. The nitrogen content can also vary within a range of $0.001 \mathrm{mg} / \mathrm{L}$ to $1.0-2.0 \mathrm{mg} / \mathrm{L} \mathrm{[1].}$ 
Most of the small lakes are shallow with plenty of light and nutrients; hence, they are among the most productive ecosystems on Earth [4]. A high level of nutrients affects the development of aquatic macrophytes, which provide refuges for planktonic invertebrates (for example, for species of the genus Daphnia sp.) from fish predation [1,5,6]. In some landscapes, especially in regions with developed agriculture, small water bodies are considered near-pristine [7]. In contrast, larger water bodies with larger catchment areas are vulnerable to pollution through intensive use and pollution of the surrounding land areas.

The mentioned above features of small water bodies, including variability of hydrochemical parameters, the heterogeneity of the habitat, and a low level of anthropogenic impact, create the basis for conserving the high biodiversity of aquatic communities (macroand micro-invertebrates, macrophytes and amphibians) of small water bodies [8,9]. For example, 134 species were found in zooplankton of two small water bodies of Poland [10]. Over 500 taxa of macroinvertebrates were recorded in 792 small water bodies in Ireland $[7,11]$. Two hundred thirty species of macroinvertebrates identified in 25 small water bodies in southern England [12].

Small water bodies are specific habitat to some particular species of planktonic invertebrates. For example, typical species of small water bodies are planktonic crustaceans Sinodiaptomus (Sinodiaptomus) sarsi (Rylov) [13], species of the genus Thermocyclops [14,15] and Diacyclops sp. [16]. Two new species of the order Calanoida (Arctodiaptomus (Arctodiaptomus) naursumensis Stepanova [17] and Gigantodiaptomus irtyshensis sp. nova [18] discovered in small lakes of East Kazakhstan. In addition to their significant role in the conservation of biodiversity [19-21], small water bodies are exemplary (model) objects for studying the adaptation of aquatic organisms to sharp fluctuations of the aquatic environment (primarily changes in salinity, temperature, and nutrient content) [22].

In contrast to large water bodies with stable habitat conditions and a relatively constant aquatic community structure [23], small water bodies are susceptible to external factors fluctuations [1]. Hence, the species composition and structure of aquatic communities can change significantly over a short time [24]. In turn, research of biological communities' response as an adaptation to fluctuations of external factors can be used for bioindication of the ecological state of small water bodies $[25,26]$. The topicality of the study of small water bodies recognized relatively recently [2]. It is, for this reason, there are relatively few articles devoted to the study of the aquatic microflora and microfauna of this category of water bodies in different regions of the world $[1,2,27,28]$. Kazakhstan is not an exception since here hydrobiological studies mainly cover large water bodies (Caspian Sea, Aral Sea, Lake Balkhash, Ile River) [23,29-34]. Significantly fewer publications focused on the study of the hydrobiological regime of small water bodies [35-37]. In this regard, the purpose of the research is to study the structure of zooplankton of small lakes in South-East Kazakhstan in connection with various physicochemical parameters to understand the role of biological variables in assessing the ecological state of aquatic ecosystems.

\section{Materials and Methods}

\subsection{Description of Study Area}

The surveyed lakes are situated at the lower altitude and partly mountainous parts of South-East Kazakhstan (Figure 1) in the arid climatic zone. The average January temperature is about $8{ }^{\circ} \mathrm{C}$ and warms up to $25^{\circ} \mathrm{C}$ in July in mountainous areas. The average annual precipitation is $500-1600 \mathrm{~mm}$ [38]. Winters are moderately warm with thaws up to $+10^{\circ} \mathrm{C}$ and frosts to $-15{ }^{\circ} \mathrm{C}$, sometimes to $-30{ }^{\circ} \mathrm{C}$ at lower altitudes. In summer, temperatures exceed $+32{ }^{\circ} \mathrm{C}$. The average annual amount of precipitation ranges from 250 to $300 \mathrm{~mm}$ at lower altitudes [39]. 


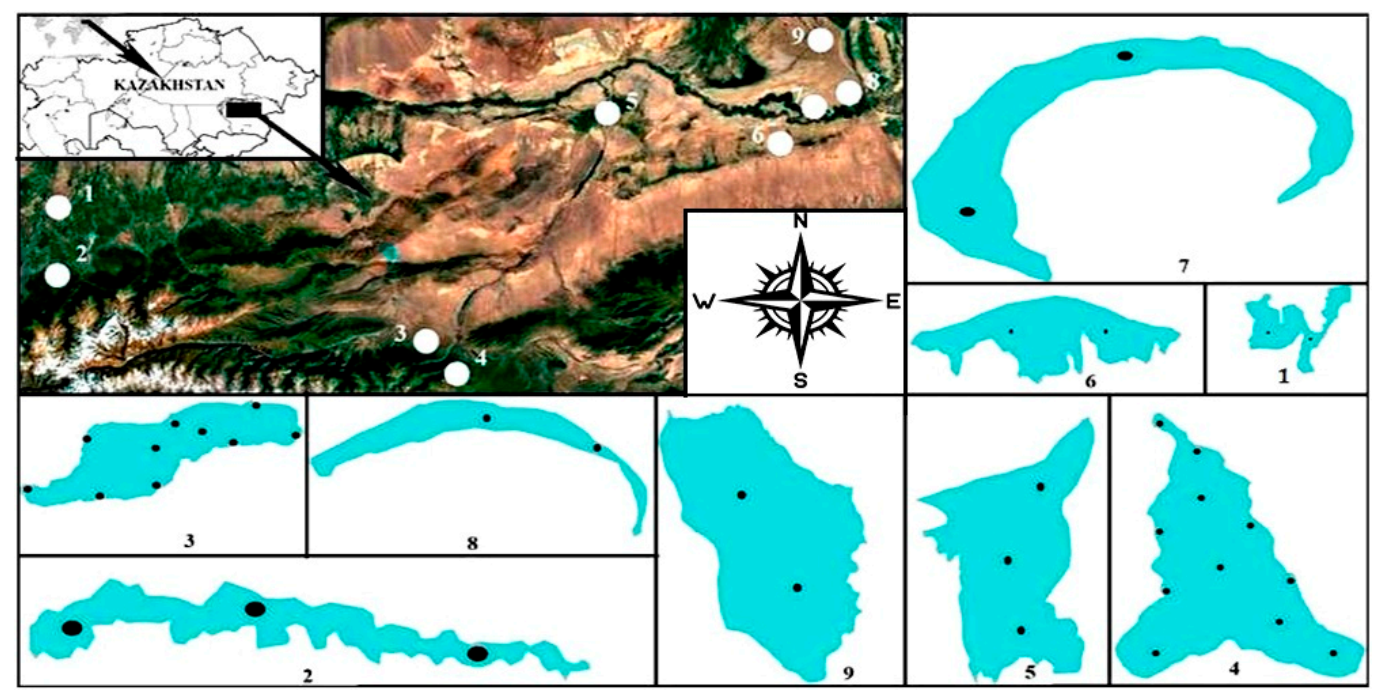

Figure 1. Map—scheme of small lakes in South-East Kazakhstan: 1-Ali, 2-Pervomaika, 3-Lower Kolsay, 4-Middle Kolsay, 5-Derevyannoe, 6-Kosagash, 7-Bolshaya Podkova, 8-Malaya Podkova, 9-Altynkol.

Lower Kolsay and Middle Kolsay Lakes are located in Kungey Alatau mountain; the rest of the lakes are at lower altitude. All of them belong to the Ili-Balkhash water basin. The surveyed lakes have a small water surface (Table 1). Lower Kolsay and Middle Kolsay are the deepest, with high transparency and low water temperatures. The river of the same name feeds them. Macrophytes do not develop. Among the plain lakes, Derevyannoe and Pervomaika are the deepest. All lakes at lower altitude are warm, with relatively low water transparency, are overgrown with macrophytes to varying degrees. Their water supply sources are groundwater, except for Lake Pervomayka, which is filled with water from the small river Karateren.

Table 1. Physical and geographical characteristics of small lakes in South-East Kazakhstan, summer 2019.

\begin{tabular}{|c|c|c|c|c|c|c|c|c|}
\hline $\begin{array}{l}\text { Lake } \\
\text { Name }\end{array}$ & $\begin{array}{l}\text { Altitude } \\
\text { above Sea } \\
\text { Level, m }\end{array}$ & $\begin{array}{l}\text { Length, } \\
\text { km }\end{array}$ & $\begin{array}{l}\text { Maximum } \\
\text { Width, km }\end{array}$ & $\begin{array}{c}\text { Water } \\
\text { Area, } \text { km }^{2}\end{array}$ & $\begin{array}{c}\text { Maximum } \\
\text { Depth, m }\end{array}$ & $\begin{array}{c}\text { Tempe- } \\
\text { rature, } \\
{ }^{\circ} \mathrm{C}\end{array}$ & Secchi, m & $\begin{array}{c}\text { Macro- } \\
\text { phyte } \\
\text { Cover, \% }\end{array}$ \\
\hline Ali & 557.0 & 0.2 & 0.2 & 0.2 & 4.0 & 24.0 & 2.0 & 50.0 \\
\hline Pervomaika & 672.0 & 3.0 & 0.2 & 0.2 & 6.0 & 24.0 & 2.0 & 50.0 \\
\hline Derevyannoe & 522.0 & 1.2 & 0.8 & 0.8 & 10.0 & 23.0 & 2.0 & 30.0 \\
\hline Altynkol & 639.0 & 0.9 & 0.4 & 0.4 & 4.0 & 24.0 & 2.0 & 30.0 \\
\hline Kosagash & 623.0 & 1.0 & 0.3 & 0.3 & 4.0 & 24.0 & 1.0 & 30.0 \\
\hline $\begin{array}{l}\text { Malaya } \\
\text { Podkova }\end{array}$ & 528.0 & 2.8 & 0.2 & 0.2 & 3.5 & 27.0 & 2.0 & 30.0 \\
\hline $\begin{array}{l}\text { Bolshaya } \\
\text { Podkova }\end{array}$ & 529.0 & 3.7 & 0.4 & 0.4 & 2.5 & 24.0 & 1.0 & 35.0 \\
\hline $\begin{array}{l}\text { Lower } \\
\text { Kolsay }\end{array}$ & 2257.0 & 1.6 & 0.4 & 0.4 & 36.0 & 16.0 & 5.0 & 0.0 \\
\hline $\begin{array}{l}\text { Middle } \\
\text { Kolsay }\end{array}$ & 2331.0 & 1.1 & 0.7 & 0.7 & 51.0 & 13.0 & 4.0 & 0.0 \\
\hline
\end{tabular}

\subsection{Field Sampling}

The lakes were surveyed in June and August 2019 on 2 to 11 sampling sites (Figure 1), depending on the lake depth. A total of 37 sampling sites installed, including in The Middle Kolsay - 11, The Lower Kolsay-10, in Derevyannoe lake-3, Pervomayka-3, and two sampling sites per lake Bolshaya Podkova, Malaya Podkova, Ali, Altynkol and Kosagash placed. The temperature, $\mathrm{pH}$ values, and dissolved oxygen were determined at each sampling site using Horiba U-50 equipment (Horiba, Ltd., Kyoto, Japan). Transparency was determined using a Secchi disk. Macrophyte cover was assessed visually. 
In order to characterize the habitat conditions of zooplankton, water samples for nutrients, total dissolved solids (TDS), and permanganate index were taken. Samples for determining nutrients were taken in glass bottles with a volume of $0.5 \mathrm{~L}$ and fixed with $1 \mathrm{~mL}$ of chloroform. Water samples for determination of the permanganate index (PI) were collected in glass containers with a volume of $0.250 \mathrm{~mL}$ and fixed with pure sulfuric acid in a 1:3 dilution. The samples were stored in a refrigerated place until they were delivered to the laboratory.

A sampling of zooplankton was carried out along with the collection of hydrochemical samples at the same sampling sites. Zooplankton was collected using a Juday plankton net (mesh size $30 \mu \mathrm{m}$ ) by pulling it from the bottom to the surface. The filtered water was poured into plastic containers with $250 \mathrm{~mL}$ and fixed with $40 \%$ formalin solution.

In order to characterize the feeding conditions of zooplankton, phytoplankton samples collected. Integrated phytoplankton samples [40] were taken from 13 sampling sites. Three samples were taken from the mountain Lake Lower Kolsay and three samples from the Lake Middle Kolsay. One phytoplankton sample per one low altitude lake collected. Phytoplankton samples were fixed with $40 \%$ formalin solution.

\subsection{Laboratory Processing}

Standard methods were used to analyze the hydrochemical parameters [41,42]. Samples of water were analyzed in three or four replicates. The nitrite nitrogen, nitrate nitrogen, ammonium nitrogen determined using a spectrophotometric method. According to the type of analysis, Griss's or Nessler's reagents, ammonium molybdate in combination with ascorbic or sulfosalicylic acid were used. The permanganate index (PI) determination was carried out using the Kubel method in acidic conditions.

Processing phytoplankton and zooplankton samples performed according to the literature $[40,43]$. The species identification of planktonic algae was carried out according to the guides [44-49]. After the sampling, phytoplankton samples were kept in the dark for 3-4 days. The water above the sediment was sucked off with a siphon through a fine sieve to a volume of $100 \mathrm{~cm}^{3}$. Before secondary settling in the dark (2-3 days), the samples poured into graduated cylinders. After settling, their volume concentrated to $5-10 \mathrm{~cm}^{3}$ with a siphon. The samples were poured into penicillin vials and fixed with one or two drops of $40 \%$ formalin. A Goryaev cell with a bottom area of $1 \mathrm{~cm}^{2}$ and a volume of $0.9 \mathrm{~mm}^{3}$ used to calculate phytoplankton abundance. The abundance of algae cells recalculated per $1 \mathrm{~m}^{3}$ according to the formula:

$$
\mathrm{N}=\frac{\mathrm{n} \times \mathrm{v} \times 10^{-6}}{\mathrm{w}}
$$

where: $\mathrm{N}$-number of cells per $1 \mathrm{~m}^{3}$ of water; $\mathrm{n}$-number of cells in a $1 \mathrm{~cm}^{3}$ Goryaeva cell; $\mathrm{v}$ - a volume of concentration, $\mathrm{cm}^{3} ; \mathrm{w}-$ a volume of water, $\mathrm{cm}^{3}$.

The biomass of each type of algae was calculated by multiplying the number of cells by its biovolumes. Thirty individuals of each species were measured to obtain their biovolumes. The specific weight of individuals is taken as 1. Total biomass of phytoplankton in the sample calculated by summarizing each species [43].

Zooplankton identified according to the species guides [13,50-53]. In each sample, the number of individuals of each species was counted using stereomicroscopes MBS-10 and MC-300 (Lytkarino Optical Glass Plant, Lytkarino, Russia). The collected sample concentrated to a volume of $150-400 \mathrm{~cm}^{3}$. After thorough mixing, three portions of the sample were taken from the sample using a $1 \mathrm{~mL}$ stamp-pipette. In this sub-sample, all recorded individuals and age stages of certain species (the most numerous) were counted in Bogorov counting chamber [40]. Bogorov counting chamber looks like a glass plate. It separated by counting chambers. These chambers hold a small volume of sample for observation under a microscope. After that, the sample was concentrated to the volume $125-150 \mathrm{~cm}^{3}$. Three sub-samples were retaken from it, in which younger stages or rare species were counted. The whole procedure was repeated once more, while the sample was concentrated to a volume of $50 \mathrm{~cm}^{3}$. The abundance of rare species was 
estimated by assessing the entire sample. For Copepoda, adult females, females with eggs, males, copepodites stages at 1-3 and 4-5 stages, and nauplii were separately counted and measured. For cladocerans were counted females with eggs or juveniles in a brood pouch, sterile females, males, and juveniles counted and measured. For each crustacean species, the abundance and mass of all stages of growth were summarized. Individual biomass was calculated using length-weight relationships [40].

Further, the abundance of individuals and the biomass of all species were summarized. The results of counting individuals are recalculated per $1 \mathrm{~m}^{3}$ using the formula $[40,43]$ (separately for each sample dilution):

$$
\mathrm{N}=\frac{\mathrm{n} \times\left(\frac{\mathrm{V} 1}{\mathrm{~V} 2}\right)}{\mathrm{V} 3}
$$

where: $\mathrm{N}$ is the abundance (ind. $/ \mathrm{m}^{3}$ ), $\mathrm{n}$ is the number of individuals in a portion (specimens), V1 is the dilution volume $\left(\mathrm{cm}^{3}\right), \mathrm{V} 2$ is the subsample volume $\left(\mathrm{cm}^{3}\right), \mathrm{V} 3$ is the filtered water volume $\left(\mathrm{m}^{3}\right)$.

The filtered volume of water was calculated by the formula:

$$
\mathrm{V} 3=\mathrm{h} \times \pi \mathrm{r}^{2}
$$

where: $\mathrm{h}$ is the length of the net pulling (water column height), and $\mathrm{r}$ is the radius of the inner ring of the Juday net.

The number of species per sample, an average individual mass of an organism, and Snannon diversity index were calculated to describe the zooplankton structure. An average individual mass of an organism (mg) was calculated as the total biomass divided by the total abundance of zooplankton for each sample. Shannon index was calculated both based on the abundance and the biomass of species in the sample [54,55] using Primer 6 Software (https: / / primer.software.informer.com/6.0/, accessed on 5 February 2021) [56]. The first version of the index is designated as Shannon $\mathrm{Ab}$ (bit/ind.), the second one as Shannon $\mathrm{Bi}$ (bit/mg) for the convenience of distinguishing them.

\subsection{Statistical Analysis}

We visualized the differences in chemical variables between samples by building a dendrogram basing on the Bray-Curtis distance. Bray-Curtis provides a measure of the differences in chemical variables between samples. Bray-Curtis Cluster Analysis was done using BioDiversityPro software [57]. Similarity level was significant only when similarity reached more than $50 \%$.

The calculation of species similarity was performed as the network analysis in JASP 0.9.0.0 (Jeffrey's Amazing Statistics Program, University of Amsterdam, Amsterdam, The Netherlands) on the botnet package in R-Statistica (R Core Team, Vienna, Austria). JASP plot analysis was created as a calculation result on the $50 \%$ similarity, level was significant only when $p<0.05$ [58]. The Redundancy Analysis (RDA) was used to identify the main factors that affect zooplankton. It was performed using the statistical software Canoco 5 (Microcomputer Power, Ithaca, NY, USA) [59].

\section{Results}

\subsection{Chemical Characteristics of Lakes}

According to the chemical data (Table 2) and their comparison with the classification [60], the water of the mountains lakes was ultra-fresh, and the lakes at a lower altitude were fresh (Table 2). Ultra-fresh water is water containing less than $0.2 \mathrm{~g} / \mathrm{dm}^{3}$ of dissolved solids, whereas fresh water is water containing less than 0.2 or $0.5 \mathrm{~g} / \mathrm{dm}^{3}$ of dissolved solids [60]. The highest values of the permanganate index are recorded in lakes at a lower altitude and the lowest one in mountains lakes. The nitrite concentration varied from 0.001 to $0.270 \mathrm{mg} / \mathrm{dm}^{3}$, with a maximum in the lakes at a lower altitude. On the contrary, the 
highest nitrate content $\left(1.30 \mathrm{mg} / \mathrm{dm}^{3}\right)$ but low $\mathrm{PO}_{4}$ concentration were recorded in the mountain's lakes. Low concentrations of $\mathrm{NH}_{4}$ recorded in the lakes at a lower altitude.

Table 2. Physical and chemical variables of small lakes in South-East Kazakhstan, mean values with standard deviation, summer 2019.

\begin{tabular}{|c|c|c|c|c|c|c|c|c|}
\hline \multirow{2}{*}{ Lake } & \multirow{2}{*}{$\begin{array}{c}\text { Mo-nth } \\
*\end{array}$} & \multirow{2}{*}{$\begin{array}{c}\text { TDS } \\
\mathrm{mg} / \mathrm{dm}^{3}\end{array}$} & \multirow{2}{*}{$\mathrm{pH}$} & \multirow{2}{*}{$\begin{array}{c}\text { PI } \\
\mathrm{mg} \mathrm{O} / \mathrm{dm}^{3}\end{array}$} & \multicolumn{4}{|c|}{ Concentration, $\mathrm{mg} / \mathrm{dm}^{3}$} \\
\hline & & & & & $\mathrm{NO}_{2}-\mathrm{N}$ & $\mathrm{NO}_{3}-\mathrm{N}$ & $\mathrm{NH}_{4}-\mathrm{N}$ & $\mathrm{PO}_{4}$ \\
\hline \multirow{2}{*}{ Ali } & 1 & $419.4 \pm 15.0$ & $7.3 \pm 0.1$ & $10.2 \pm 0.1$ & $0.001 \pm 0.001$ & $0.7 \pm 0.1$ & $0.07 \pm 0.01$ & $0.07 \pm 0.01$ \\
\hline & 2 & $395.8 \pm 12.0$ & $7.8 \pm 0.1$ & $8.4 \pm 0.01$ & $0.001 \pm 0.001$ & $0.3 \pm 0.1$ & $0.01 \pm 0.00$ & $0.09 \pm 0.01$ \\
\hline \multirow{2}{*}{ Pervomaika } & 1 & $434.0 \pm 15.5$ & $7.5 \pm 0.1$ & $10.1 \pm 0.1$ & $0.030 \pm 0.001$ & $0.6 \pm 0.4$ & $0.06 \pm 0.01$ & $0.07 \pm 0.01$ \\
\hline & 2 & $511.6 \pm 16.8$ & $7.7 \pm 0.1$ & $10.1 \pm 0.1$ & $0.030 \pm 0.001$ & $0.7 \pm 0.2$ & $0.01 \pm 0.00$ & $0.10 \pm 0.02$ \\
\hline \multirow{2}{*}{ Derevyannoe } & 1 & $576.4 \pm 16.2$ & $7.3 \pm 0.1$ & $9.2 \pm 0.2$ & $0.006 \pm 0.001$ & $0.4 \pm 0.2$ & $0.01 \pm 0.00$ & $0.04 \pm 0.01$ \\
\hline & 2 & $571.3 \pm 16.7$ & $8.0 \pm 0.1$ & $7.5 \pm 0.1$ & $0.007 \pm 0.001$ & $0.6 \pm 0.4$ & $0.01 \pm 0.00$ & $0.03 \pm 0.01$ \\
\hline \multirow{2}{*}{ Altynkol } & 1 & $292.9 \pm 10.7$ & $7.6 \pm 0.1$ & $9.4 \pm 0.2$ & $0.270 \pm 0.020$ & $0.3 \pm 0.1$ & $0.001 \pm 0.000$ & $0.10 \pm 0.01$ \\
\hline & 2 & $260.3 \pm 10.7$ & $7.9 \pm 0.1$ & $9.6 \pm 0.2$ & $0.015 \pm 0.001$ & $0.6 \pm 0.4$ & $0.005 \pm 0.003$ & $0.13 \pm 0.02$ \\
\hline \multirow{2}{*}{ Kosagash } & 1 & $331.9 \pm 11.3$ & $7.0 \pm 0.1$ & $9.6 \pm 0.2$ & $0.010 \pm 0.001$ & $0.4 \pm 0.2$ & $0.005 \pm 0.003$ & $0.18 \pm 0.02$ \\
\hline & 2 & $346.8 \pm 10.9$ & $8.0 \pm 0.1$ & $8.5 \pm 0.1$ & $0.130 \pm 0.010$ & $0.8 \pm 0.6$ & $0.011 \pm 0.002$ & $0.08 \pm 0.01$ \\
\hline & 1 & $302.8 \pm 10.4$ & $7.8 \pm 0.1$ & $9.1 \pm 0.2$ & $0.015 \pm 0.001$ & $0.3 \pm 0.1$ & $0.041 \pm 0.004$ & $0.15 \pm 0.02$ \\
\hline Podkova & 2 & $403.0 \pm 14.2$ & $7.9 \pm 0.1$ & $10.2 \pm 0.1$ & $0.007 \pm 0.001$ & $0.3 \pm 0.1$ & $0.001 \pm 0.001$ & $0.13 \pm 0.02$ \\
\hline Bolshaya & 1 & $319.2 \pm 11.3$ & $7.0 \pm 0.1$ & $8.9 \pm 0.1$ & $0.007 \pm 0.001$ & $0.3 \pm 0.1$ & $0.005 \pm 0.003$ & $0.30 \pm 0.20$ \\
\hline Podkova & 2 & $429.8 \pm 15.2$ & $7.0 \pm 0.1$ & $10.2 \pm 0.1$ & $0.010 \pm 0.002$ & $0.4 \pm 0.2$ & $0.001 \pm 0.001$ & $0.09 \pm 0.02$ \\
\hline Lower & 1 & $188.8 \pm 2.0$ & $7.9 \pm 0.2$ & $0.9 \pm 0.1$ & $0.008 \pm 0.001$ & $1.3 \pm 0.4$ & $0.014 \pm 0.003$ & $0.02 \pm 0.01$ \\
\hline Kolsay & 2 & $188.6 \pm 2.0$ & $8.3 \pm 0.1$ & No data & $0.002 \pm 0.001$ & $0.1 \pm 0.0$ & $0.010 \pm 0.003$ & $0.08 \pm 0.01$ \\
\hline Middle & 1 & $70.0 \pm 0.4$ & $7.3 \pm 0.1$ & $0.8 \pm 0.1$ & $0.031 \pm 0.002$ & $1.1 \pm 0.3$ & $0.38 \pm 0.02$ & $0.019 \pm 0.001$ \\
\hline Kolsay & 2 & $184.6 \pm 1.9$ & $8.0 \pm 0.1$ & No data & $0.001 \pm 0.001$ & $0.1 \pm 0.0$ & $0.009 \pm 0.007$ & $0.030 \pm 0.002$ \\
\hline
\end{tabular}

${ }^{*}$ Notice. 1-June, 2-August.

Despite the significant variability of chemical parameters in the surveyed small lakes, Bray-Curtis cluster analysis did not reveal statistically significant differences between the nutrient content (Figure 2).

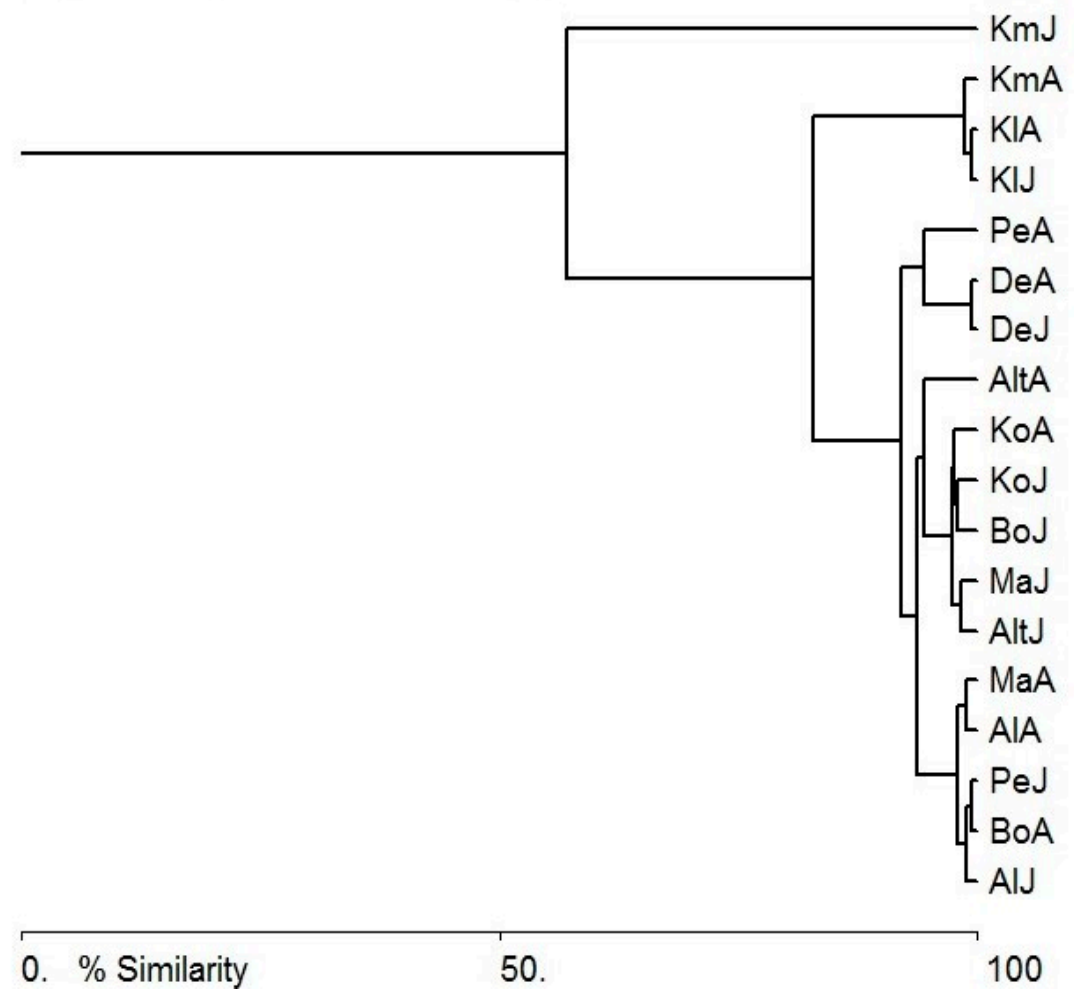

Figure 2. Chemical variables similarity assessment according to the Bray-Curtis cluster analysis. 
Abbrevations: AlJ—Ali June, AlA—Ali August, PeJ-Pervomaika June, PeA—Pervomaika August, DeJ-Derevyannoe June, DeA—Derevyannoe August, AltJ—Altynkol June, AltA—Altynkol August, KoJ—Kosagash June, KoA—Kosagash August, MaJ—Malaya Podkova June, MaA—Malaya Podkova August, BoJ-Bolshaya Podkova June, BoA-Bolshaya Podkova August, KoJ-Lower Kolsay June, KoA-Lower Kolsay August, KoJ-Middle Kolsay June, KoA-Middle Kolsay August. The line between lakes reflects similarity.

\subsection{Phytoplankton}

Phytoplankton of the surveyed lakes was represented by 74 taxa from 6 divisions Bacillariophyta - 37, Chlorophyta-17, Miozoa-4, Cyanobacteria-9, Euglenozoa-6, Charophyta-1 (Table 3). The lowest number of species (5) was recorded in the Middle Kolsay, the highest in August in Lake Kosagash-29. In all surveyed lakes, only one species of diatoms was found-Cymbella lanceolata (C. Agardh) Kirchner.

Table 3. Taxonomic composition of phytoplankton in small lakes in South-East Kazakhstan, summer 2019.

\begin{tabular}{|c|c|c|c|c|c|c|c|c|c|}
\hline \multirow{2}{*}{ Taxon Name } & \multicolumn{9}{|c|}{ * Lakes } \\
\hline & 1 & 2 & 3 & 4 & 5 & 6 & 7 & 8 & 9 \\
\hline \multicolumn{10}{|c|}{ Bacillariophyta } \\
\hline $\begin{array}{c}\text { Encyonema leibleinii (C.Agardh) W.J.Silva, R.Jahn, } \\
\text { T.A.V.Ludwig, \& M.Menezes }\end{array}$ & + & + & - & - & - & - & - & - & - \\
\hline Cyclotella ocellata Pantocsek & - & - & - & - & - & - & - & + & + \\
\hline Cymbella lanceolata (C. Agardh) Kirchner & + & + & + & + & + & + & + & + & + \\
\hline Navicula minima Grunow & + & - & - & - & + & - & - & - & - \\
\hline Navicula radiosa Kützing & + & - & - & - & - & - & - & - & - \\
\hline Aneumastus tusculus (Ehrenberg) D.G.Mann \& A.J.Stickle & + & - & - & - & - & - & - & - & - \\
\hline Ulnaria acus (Kützing) Aboal in Aboal & + & + & + & + & + & + & + & - & - \\
\hline Odontidium hyemale (Roth) Kützing & - & - & - & - & - & - & - & + & + \\
\hline Diatoma vulgaris Bory & + & + & - & + & + & - & + & - & - \\
\hline Amphora ovalis (Kützing) Kützing & - & - & + & + & + & + & - & - & + \\
\hline Achnanthidiumminutissimum (Kützing) Czarnecki & - & - & - & - & - & - & - & - & + \\
\hline Cyclotella meneghiniana Kützing & - & - & + & - & - & + & - & + & + \\
\hline Cyclotella kuetzingiana Thwaites & - & - & - & - & - & - & - & + & + \\
\hline Cyclotella planctonica Brunnthaler & - & - & - & - & - & - & - & - & + \\
\hline Cyclotella sp. & - & - & - & - & - & - & - & - & + \\
\hline $\begin{array}{l}\text { Gomphonema acuminatum var. longiceps (Ehrenberg) } \\
\text { N.Abarca \& R.Jahn }\end{array}$ & - & - & - & - & - & - & - & + & - \\
\hline Cocconeis placentula Ehrenberg & - & - & - & - & - & - & - & - & + \\
\hline Fragilaria capucina var. vaucheriae (Kützing) Lange-Bertalot & - & - & - & - & - & - & - & - & + \\
\hline Cymbella ventricosa (C.Agardh) C.Agardh & - & - & - & - & - & - & - & - & + \\
\hline Lindavia kurdica (Håkansson) T.Nakov & - & - & + & - & - & + & + & + & + \\
\hline Aulacoseira granulata (Ehrenberg) Simonsen & - & - & + & - & - & - & - & - & - \\
\hline Epithemia gibba (Ehrenberg) Kützing & - & + & + & + & + & - & - & - & - \\
\hline Mastogloia elliptica (C.Agardh) (C.Agardh) Cleve & - & - & - & + & - & - & - & - & - \\
\hline Navicula rhynchocephala Kützing & - & - & - & + & - & + & - & - & - \\
\hline $\begin{array}{c}\text { Synedra capitata var. gracilis Poretzky ex } \\
\text { Proschkina-Lavrenko }\end{array}$ & - & - & - & + & - & - & - & - & - \\
\hline Synedra ulna var. ulna (Nitzsch) Ehrenberg & - & - & - & - & + & - & + & + & - \\
\hline Gomphonema constrictum Ehrenberg & - & - & - & - & + & - & - & - & - \\
\hline Eunotia lunaris (Ehrenberg) Grunow & - & - & - & - & + & - & - & - & - \\
\hline Urosolenia longiseta (O.Zacharias) Edlund \& Stoermer & - & - & - & - & + & - & - & - & - \\
\hline Craticula ambigua (Ehrenberg) D.G.Mann & - & - & - & + & - & + & - & - & - \\
\hline Navicula oblonga (Kützing) Kützing & - & - & - & - & - & + & - & - & - \\
\hline Navicula exigua W.Gregory & - & - & - & - & - & - & - & - & + \\
\hline Nitzschiapalea (Kützing) W.Smith & - & - & - & - & - & + & - & - & - \\
\hline Gomphonema sphaerophorum Ehrenberg & - & - & - & - & - & - & - & - & + \\
\hline Meridioncirculare (Greville) C.Agardh & - & - & - & - & - & - & - & - & + \\
\hline Achnanthes sp. & - & - & - & - & + & - & - & - & - \\
\hline Asterionella formosa Hassall & - & - & - & - & - & - & - & - & + \\
\hline
\end{tabular}


Table 3. Cont.

\begin{tabular}{|c|c|c|c|c|c|c|c|c|c|}
\hline \multirow{2}{*}{ Taxon Name } & \multicolumn{9}{|c|}{ * Lakes } \\
\hline & 1 & 2 & 3 & 4 & 5 & 6 & 7 & 8 & 9 \\
\hline \multicolumn{10}{|c|}{ Chlorophyta } \\
\hline Nephrocytium lunatum West & + & + & - & - & + & - & - & - & - \\
\hline Kirchneriella lunaris (Kirchner) Möbius & + & - & - & + & - & - & - & - & - \\
\hline Monoraphidium contortum (Thuret) Komárková-Legnerová & + & - & + & + & + & + & - & - & - \\
\hline Scenedesmus bijugatus var. bijugatus Kützing & + & + & + & + & + & + & + & - & - \\
\hline Tetraëdron minimum (A.Braun) Hansgirg & + & + & - & + & + & - & + & - & - \\
\hline S. quadricauda var. quadricauda (Turpin) Brébisson & - & + & + & + & + & + & - & - & - \\
\hline Scenedesmus arcuatus (Lemmermann) Lemmermann & + & - & - & - & - & - & - & - & - \\
\hline Staurastrum tetracerum Ralfs ex Ralfs & + & + & + & - & + & + & - & - & - \\
\hline Monoraphidium minutum (Nägeli) Komárková-Legnerová & + & - & - & + & + & - & - & - & - \\
\hline Tetraëdron minutissimum Korshikov & - & + & - & + & + & + & - & - & - \\
\hline Closteriopsis longissima (Lemmermann) Lemmermann & - & + & - & + & + & - & - & + & - \\
\hline Ankistrodesmus densus Korshikov & - & - & - & - & - & - & + & - & - \\
\hline Sphaerocystis planctonica (Korshikov) Bourrelly & - & - & - & - & - & - & - & + & - \\
\hline Coelastrum microporum Nägeli & - & + & - & + & - & - & - & - & - \\
\hline Monactinus simplex (Meyen) Corda & - & - & + & - & - & - & - & - & - \\
\hline Coelastrum microporum Nägeli & - & - & - & + & - & - & - & - & - \\
\hline Pediastrum duplex Meyen & - & - & - & + & - & - & - & - & - \\
\hline \multicolumn{10}{|c|}{ Charophyta } \\
\hline \multicolumn{9}{|c|}{$\stackrel{+}{+}^{-}$} & - \\
\hline Snowella rosea (J.W.Snow) Elenkin & - & + & - & - & - & - & - & - & - \\
\hline Merismopedia tranquilla (Ehrenberg) Trevisan & - & - & + & + & + & + & + & - & - \\
\hline Microcystis flosaquae (Wittrock) Kirchner & - & - & + & - & - & - & - & - & - \\
\hline Gomphosphaeria aponina Kützing & - & - & - & + & - & + & - & - & - \\
\hline Snowella lacustris (Chodat) Komárek \& Hindák & - & - & + & + & - & + & - & - & - \\
\hline $\begin{array}{c}\text { Anathece clathrata (West \& G.S.West) Komárek, Kastovsky \& } \\
\text { Jezberová }\end{array}$ & - & - & - & - & - & - & - & + & - \\
\hline Oscillatoria sp. & + & - & - & - & - & - & - & - & - \\
\hline Microcystis pulverea f. raceformis (Nygaard) Hollerbach & - & - & - & + & + & + & - & - & - \\
\hline Anabaena flosaquae Brébisson ex Bornet \& Flauhault 66 & - & - & - & - & + & - & - & - & - \\
\hline \multicolumn{10}{|l|}{ 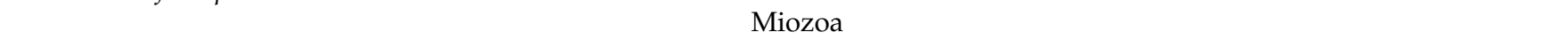 } \\
\hline Peridiniopsis quadridens (F.Stein) Bourrelly & + & + & + & + & + & + & + & - & - \\
\hline Peridinium cinctum (O.F.Müller) Ehrenberg & - & + & - & + & - & - & + & - & - \\
\hline Ceratium hirundinella (O.F.Müller) Dujardin & + & - & - & + & + & + & + & - & - \\
\hline Kolkwitziella acuta (Apstein) Elbrächter & - & + & + & + & - & - & + & - & - \\
\hline \multicolumn{10}{|c|}{ Euglenozoa } \\
\hline Lepocinclis fusiformis (H.J.Carter) Lemmermann & - & - & - & + & - & - & - & - & - \\
\hline Lepocinclis acus (O.F.Müller) B.Marin \& Melkonian & - & - & + & - & + & - & - & - & - \\
\hline Monomorphinapyrum (Ehrenberg) Mereschkowsky & - & - & - & - & + & - & - & - & - \\
\hline Phacus curvicauda Svirenko & - & + & - & - & + & - & - & - & - \\
\hline Phacus caudatus Hübne & - & - & + & - & - & - & - & - & - \\
\hline Euglenaviridis (O.F.Müller) Ehrenberg & - & - & - & - & + & - & - & - & - \\
\hline
\end{tabular}

${ }^{*}$ Notice. 1-Ali, 2-Altynkol, 3-Bolshaya Podkova, 4-Derevyannoe, 5-Kosagash, 6-Malaya Podkova, 7-Pervomaika, 8-Lower Kolsay, 9-Middle Kolsay.

According to the JASP analysis (Figure 3), algae species composition was unique in each of the lakes at a lower altitude. A high level of correlation was found only between the phytoplankton communities of Pervomaika lake in August and Ali in June, Ali and Kosagash (June), Malaya Podkova (June) and Bolshaya Podkova (June). For the lakes at lower altitude, the species composition of phytoplankton depended on the sampling time. For example, within each of the lakes Derevyannoe, Altynkol, Malaya Podkova, Ali, Bolshaya Podkova, the similarity of species composition in June and August was low. 


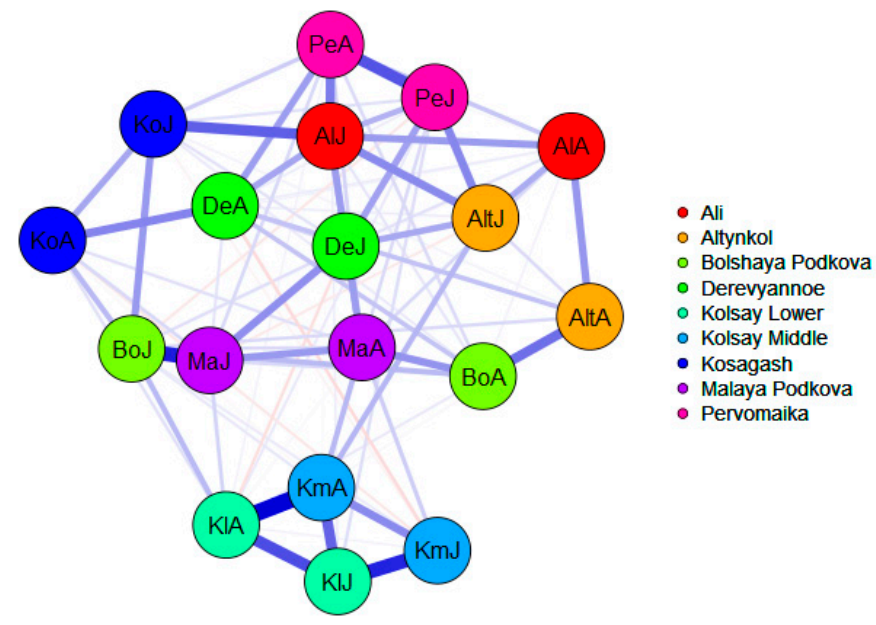

Figure 3. Phytoplankton species similarity assessment according to the JASP network plot. Abbrevations: AlJ—Ali June, AlA—Ali August, PeJ—Pervomaika June, PeA-Pervomaika August, DeJ-Derevyannoe June, DeA—Derevyannoe August, AltJ—Altynkol June, AltA—Altynkol August, KoJ—Kosagash June, KoA—Kosagash August, MaJ-Malaya Podkova June, MaA—Malaya Podkova August, BoJ-Bolshaya Podkova June, BoA-Bolshaya Podkova August, KoJ-Lower Kolsay June, KoA-Lower Kolsay August, KoJ-Middle Kolsay June, KoA-Middle Kolsay August. The line width between lakes reflects the correlation value; blue-positive, red rose-negative, the color saturation-strength of correlation.

The species composition of phytoplankton in mountain lakes was relatively constant throughout the summer, with a high similarity both within one lake and between lakes. Figure 3 demonstrates the uniqueness of microalgae species composition of mountain lakes compared to plain lakes.

The abundance of phytoplankton varied from $8.5 \times 10^{7}$ to $2.71667 \times 10^{9}$ cells $/ \mathrm{m}^{3}$ (Table 4). Phytoplankton biomass varied from 468.88 to $15,845.18 \mathrm{mg} / \mathrm{m}^{3}$. In most lakes, phytoplankton quantitative variables increased from June to August, except for Middle Kolsay Lake, where the abundance decreased on average by five times. A decrease in phytoplankton biomass from June to August was recorded in lakes Pervomaika, Kosagash and Malaya Podkova.

Table 4. The quantitative variables of phytoplankton in small lakes in South-East Kazakhstan, summer 2019.

\begin{tabular}{|c|c|c|c|c|c|}
\hline Lake & Month & $\begin{array}{c}\text { Abundance, } \\
\text { Million Cells } / \mathrm{m}^{3}\end{array}$ & $\begin{array}{l}\text { Dominant } \\
\text { Division }\end{array}$ & Biomass, $\mathrm{g} / \mathrm{m}^{3}$ & $\begin{array}{l}\text { Dominant } \\
\text { Division }\end{array}$ \\
\hline \multirow{2}{*}{ Ali } & 1 & 85.00 & Chlorophyta & 0.52 & Miozoa \\
\hline & 2 & 253.31 & Chlorophyta & 1.72 & Chlorophyta \\
\hline \multirow{2}{*}{ Pervomaika } & 1 & 195.01 & Miozoa & 15.81 & Miozoa \\
\hline & 2 & 293.31 & Miozoa & 10.50 & Miozoa \\
\hline \multirow{2}{*}{ Derevyannoe } & 1 & 223.35 & Cyanobacteria & 3.21 & Miozoa \\
\hline & 2 & 991.72 & Chlorophyta & 2.60 & Miozoa \\
\hline \multirow{2}{*}{ Altynkol } & 1 & 210.01 & Chlorophyta & 0.40 & Miozoa \\
\hline & 2 & 580.01 & Cyanobacteria & 0.51 & Bacillariophyta \\
\hline \multirow{2}{*}{ Kosagash } & 1 & 1050.01 & Chlorophyta & 2.21 & Miozoa \\
\hline & 2 & 2716.71 & Cyanobacteria & 1.61 & Chlorophyta \\
\hline \multirow{2}{*}{ Malaya Podkova } & 1 & 581.70 & Cyanobacteria & 2.01 & Bacillariophyta \\
\hline & 2 & 2716.71 & Bacillariophyta & 1.60 & Bacillariophyta \\
\hline \multirow{2}{*}{ Bolshaya Podkova } & 1 & 230.01 & Cyanobacteria & 0.51 & Bacillariophyta \\
\hline & 2 & 521.71 & Bacillariophyta & 0.90 & Bacillariophyta \\
\hline \multirow{2}{*}{ Lower Kolsay } & 1 & 1100.01 & Cyanobacteria & 4.20 & Bacillariophyta \\
\hline & 2 & 2123.30 & Bacillariophyta & 9.81 & Bacillariophyta \\
\hline \multirow{2}{*}{ Middle Kolsay } & 1 & 198.31 & Bacillariophyta & 2.40 & Bacillariophyta \\
\hline & 2 & 730.01 & Bacillariophyta & 6.30 & Bacillariophyta \\
\hline
\end{tabular}


Diatoms dominated the phytoplankton in Middle Kolsay. Miozoa (mainly Ceratium hirundinella (O.F.Müller) Dujardin) had the highest contribution to the total abundance and biomass of phytoplankton in Pervomaika. In June, cyanobacteria dominated regarding abundance, diatoms regarding biomass in mountain lake Lower Kolsay and lakes at lower altitude Malaya Podkova and Bolshaya Podkova. In August, diatoms made the main contribution to the total abundance and biomass of phytoplankton in these lakes. In other lakes, Cyanobacteria and Chlorophyta dominated regarding abundance; Miozoa by biomass.

Most often the diversity of phytoplankton in lakes at lower altitudes was high (Table 5). In almost all lakes, except Malaya Podkova, an increase in the Shannon index was observed from June to August. In mountain lakes, phytoplankton diversity was at a moderate level. The Shannon index of phytoplankton in Lower Kolsay Lake was similar during the summer, while the diversity of phytoplankton in Middle Kolsay Lake was lower (1.33-2.39) in August. According to the average weight of the cells, phytoplankton of the lakes at lower altitudes consisted of smaller species compared to the phytoplankton of mountain lakes.

Table 5. Structural variables of phytoplankton in small lakes in South-East Kazakhstan, summer 2019.

\begin{tabular}{|c|c|c|c|c|c|}
\hline Lake & Month & Species Number & Shannon Ab & Shannon Bi & $\begin{array}{c}\text { Average Weight of } \\
\text { the Cell, } \\
\times 10^{-6}, \mathrm{mg}\end{array}$ \\
\hline \multirow{2}{*}{ Ali } & June & 11 & 2.81 & 1.91 & 0.6802 \\
\hline & August & 18 & 3.69 & 2.80 & 0.7577 \\
\hline \multirow{2}{*}{ Pervomaika } & June & 10 & 2.70 & 2.76 & 1.0246 \\
\hline & August & 19 & 2.23 & 3.35 & 1.5076 \\
\hline \multirow{2}{*}{ Derevyannoe } & June & 15 & 2.98 & 3.10 & 1.0403 \\
\hline & August & 21 & 2.98 & 2.74 & 0.9210 \\
\hline \multirow{2}{*}{ Altynkol } & June & 21 & 3.31 & 1.75 & 0.5297 \\
\hline & August & 26 & 3.21 & 2.55 & 0.7955 \\
\hline \multirow{2}{*}{ Kosagash } & June & 19 & 2.05 & 2.96 & 1.4439 \\
\hline & August & 29 & 3.30 & 3.04 & 0.9220 \\
\hline \multirow{2}{*}{ Malaya Podkova } & June & 23 & 3.23 & 2.82 & 0.8721 \\
\hline & August & 12 & 0.76 & 0.39 & 0.5149 \\
\hline \multirow{2}{*}{ Bolshaya Podkova } & June & 9 & 2.21 & 0.46 & 0.2079 \\
\hline & August & 14 & 3.10 & 1.34 & 0.4333 \\
\hline \multirow{2}{*}{ Lower Kolsay } & June & 11 & 1.73 & 1.92 & 1.1062 \\
\hline & August & 7 & 1.71 & 2.22 & 1.2963 \\
\hline \multirow{2}{*}{ Middle Kolsay } & June & 15 & 2.39 & 1.60 & 0.6682 \\
\hline & August & 5 & 1.33 & 1.60 & 1.1994 \\
\hline
\end{tabular}

\subsection{Zooplankton}

Zooplankton was represented by 58 taxa, of which rotifers-32, cladocerans-15, copepods-11. The minimum number of species of planktonic invertebrates was recorded in the lake Bolshaya Podkova-2, the maximum in the Lower Kolsay Lake-23 (Table 6).

Table 6. Taxonomic composition of zooplankton in small lakes in South-East Kazakhstan, summer 2019.

\begin{tabular}{|c|c|c|c|c|c|c|c|c|c|}
\hline \multirow{2}{*}{ Taxon Name } & \multicolumn{9}{|c|}{ * Lakes } \\
\hline & 1 & 2 & 3 & 4 & 5 & 6 & 7 & 8 & 9 \\
\hline & \multicolumn{9}{|c|}{ Rotifera } \\
\hline Bdelloida gen.sp. & - & - & - & - & - & + & - & + & - \\
\hline Asplanchnabrightwelli (Gosse) & - & - & - & - & - & - & - & + & + \\
\hline Asplanchna priodonta (Gosse) & + & + & + & + & + & + & + & + & + \\
\hline Bipalpus hudsoni (Imhof) & + & - & - & + & - & + & + & - & - \\
\hline Brachionus angularis (Gosse) & - & - & - & - & + & - & - & - & - \\
\hline Brachionus plicatilis (Muller) & + & - & - & - & - & + & - & - & - \\
\hline
\end{tabular}


Table 6. Cont.

\begin{tabular}{|c|c|c|c|c|c|c|c|c|c|}
\hline \multirow{2}{*}{ Taxon Name } & \multicolumn{9}{|c|}{ * Lakes } \\
\hline & 1 & 2 & 3 & 4 & 5 & 6 & 7 & 8 & 9 \\
\hline \multicolumn{10}{|c|}{ Rotifera } \\
\hline B. quadridentatus (Hermann) & + & + & - & - & - & + & + & - & - \\
\hline B.quadridentatus brevispinus (Ehrenberg) & - & - & - & + & - & - & - & - & - \\
\hline Brachionus calyciflorus anureiformis Brehm & - & + & - & - & - & - & - & - & - \\
\hline B. calyciflorus dorcas Gosse & - & + & - & - & - & - & - & - & - \\
\hline Brachionus diversicornis (Daday) & - & - & - & + & - & - & - & - & - \\
\hline Filinia longiseta Ehren. & - & - & - & - & - & - & - & + & - \\
\hline Filinia terminalis (Plate) & - & - & - & - & - & - & - & + & - \\
\hline Conochilus dossuarius (Hudson) & - & - & - & - & - & + & - & - & - \\
\hline Keratella cochlearis (Gosse) & + & - & - & - & - & - & + & + & + \\
\hline Keratella quadrata (Muller) & - & - & - & + & - & + & - & + & + \\
\hline Platyias quadricornis (Ehrenberg) & + & - & - & - & - & - & + & - & - \\
\hline Polyarthra dolichoptera (Idelson) & + & + & - & - & - & + & + & + & - \\
\hline Polyarthra euryptera (Wierzejski) & - & - & + & - & - & - & - & - & - \\
\hline Synchaeta stylata (Wierzejski) & + & - & + & - & + & - & + & + & - \\
\hline Synchaeta tremula (Muller) & - & - & - & - & - & - & + & - & - \\
\hline Synchaeta sp. & - & - & - & - & - & + & - & + & - \\
\hline Trichocerca (Diurella) heterodactyla (Tschugunoff) & - & + & - & - & - & + & - & - & - \\
\hline Trichocerca elongata (Gosse) & - & - & - & - & - & + & - & - & - \\
\hline Trichocerca similis (Wierzejski) & - & - & + & - & - & - & - & - & - \\
\hline Trichotria pocillum (Muller) & - & - & - & - & - & - & + & - & - \\
\hline Testudinella patina (Hermann) & - & + & - & - & - & - & - & - & - \\
\hline Testudinella sp. & + & + & + & + & + & + & + & + & - \\
\hline Hexarthra oxyuris (Zernov) & - & - & - & + & - & - & - & - & - \\
\hline Lecane ungulata (Gosse) & - & - & - & + & - & - & - & - & - \\
\hline Lecane luna (Muller) & - & - & - & - & - & + & + & - & - \\
\hline Lepadella ovalis (Muller) & - & - & - & - & - & + & - & - & - \\
\hline \multicolumn{10}{|c|}{ Cladocera } \\
\hline Alona affinis Leydig & - & - & - & - & - & - & - & + & - \\
\hline Alona rectangula (Sars) & + & + & - & + & - & - & + & + & - \\
\hline Alona sp. & - & - & - & - & - & + & - & - & - \\
\hline Bosmina (Bosmina) longirostris (O.F. Muller) & + & + & + & + & + & + & + & + & - \\
\hline Camptocercus sp. & + & - & - & - & - & - & - & - & - \\
\hline Diaphanosoma brachyurum (Lievin) & - & - & + & - & - & + & - & - & - \\
\hline D. macrophtalma (Korovch. Et Mirabd.) & - & + & - & - & - & - & - & - & - \\
\hline Simocephalus vetulus (O.F.Muller) & - & - & + & - & - & - & - & - & - \\
\hline Moina micrura (Kurz) & - & + & + & + & + & - & - & - & - \\
\hline Daphnia (Daphnia) galeata (G.O. Sars) & + & - & + & - & - & - & - & + & + \\
\hline Daphnia (Daphnia) hyalina (Leydig) & - & - & + & - & - & - & - & - & - \\
\hline Daphnia (Daphnia) longispina O.F. Muller) & - & - & - & - & - & - & - & + & + \\
\hline Daphnia (Daphnia) longiremis O.F. Muller) & - & - & - & - & - & - & - & + & - \\
\hline Ceriodaphnia sp. & + & - & + & - & - & - & - & - & - \\
\hline Chydorus sphaericus (O.F. Muller) & - & - & - & - & - & - & + & + & + \\
\hline \multicolumn{10}{|c|}{ Copepoda } \\
\hline Eucyclops (s.str.) macruroides (Lilljeborg) & - & - & - & - & - & + & - & - & - \\
\hline Eucyclops serrulatus (Lilljeborg) & - & - & - & - & - & - & - & + & + \\
\hline Cyclopoida gen.sp. & + & + & + & + & + & + & + & + & + \\
\hline Cyclops vicinus (Uljanin) & - & - & - & - & - & - & - & + & + \\
\hline Macrocyclops albidus (Jurine) & - & - & - & - & - & - & - & + & \\
\hline Thermocyclops taihokuensis (Harada) & + & + & - & - & - & + & - & - & - \\
\hline Thermocyclops crassus (Fischer) & - & + & + & - & + & - & - & - & - \\
\hline Acanthodiaptomus denticornis (Wierzejski) & - & - & - & + & - & - & - & - & + \\
\hline Arctodiaptomus bacillifer (Koelbel) & - & - & - & - & + & - & - & - & - \\
\hline Diaptomidae gen.sp. & - & - & + & + & - & - & - & - & + \\
\hline Harpacticoida gen.sp. & - & - & - & - & + & - & - & - & - \\
\hline
\end{tabular}

* Notice. 1-Derevyannoe, 2-Kosagash, 3-Altynkol, 4-Malaya Podkova, 5-Bolshaya Podkova, 6-Pervomaika, 7-Ali, 8-Lower Kolsay, 9-Middle Kolsay. 
According to the JASP network plot (Figure 4), zooplankton species composition varied significantly both be-tween lakes and within the same lake but in different months. Relatively constant species composition for two months was recorded in the zooplankton of lakes Ali and Altynkol. In August, Bolshaya Podkova and Malaya Podkova lakes had a high level of similarity by zooplankton species composition, while in June, it was not significant. Zooplankton species composition remained constant during the summer in Middle Kolsay. In August, the zooplankton species composition of Lower Kolsay changed significantly. In general, the composition of zooplankton of mountain lakes differed from the zooplankton species composition of the lakes at a lower altitude.

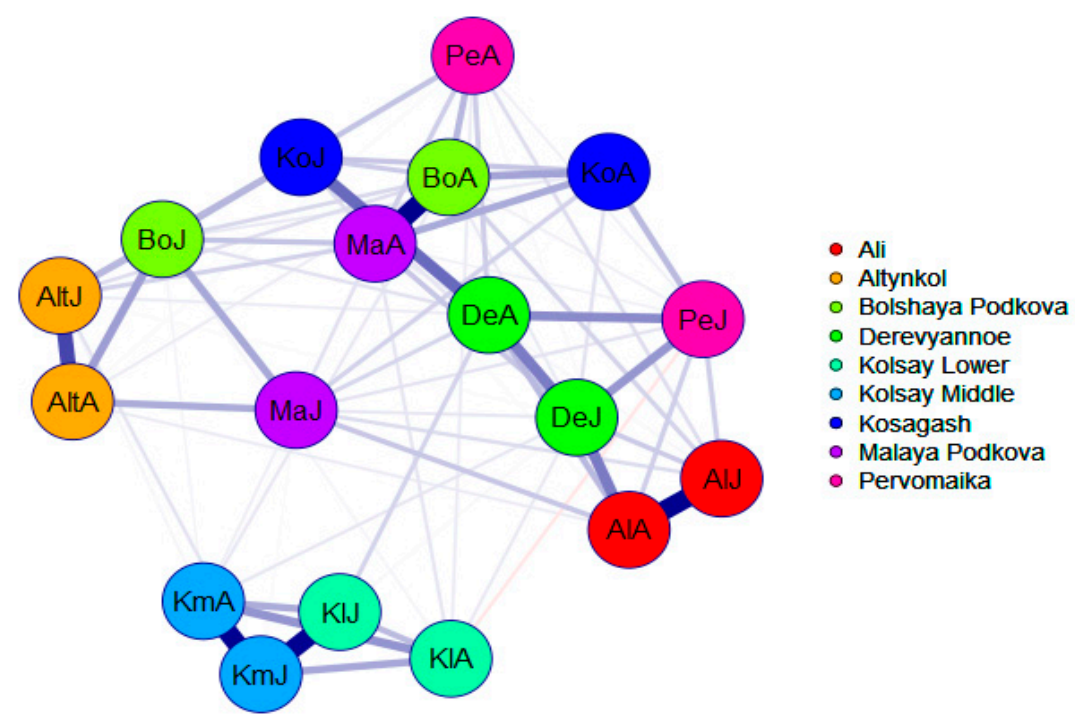

Figure 4. Zooplankton species similarity assessment according to the JASP network plot Abbrevations: AlJ—Ali June, AlA—Ali August, PeJ—Pervomaika June, PeA—Pervomaika August, DeJDerevyannoe June, DeA-Derevyannoe August, AltJ-Altynkol June, AltA—Altynkol August, KoJ-Kosagash June, KoA—Kosagash August, MaJ-Malaya Podkova June, MaA—Malaya Podkova August, BoJ-Bolshaya Podkova June, BoA-Bolshaya Podkova August, KoJ-Lower Kolsay June, KoA-Lower Kolsay August, KoJ-Middle Kolsay June, KoA-Middle Kolsay August. The line width between lakes reflects the correlation value; blue-positive, red rose-negative, the color saturation-strength of correlation.

The abundance and biomass of zooplankton fluctuated significantly across the lakes (Tables 7 and 8). The highest values of zooplankton abundance were recorded in June in Lake Bolshaya Podkova. In August the lowest value of the variable was recorded in the same lake (Table 5). A possible reason for such changes can be the fluctuations in the hydrochemical parameters of this lake. The total abundance of planktonic invertebrates increased noticeably from June to August in most lakes (except Malaya Podkova, Bolshaya Podkova, Kosagash, and Middle Kolsay).

In June, the highest values of zooplankton biomass were found in Bolshaya Podkova (Table 8). In August, zooplankton biomass of the lakes Bolshaya Podkova and Kosagash was the lowest. 
Table 7. Zooplankton abundance in small lakes of South-East Kazakhstan, mean values with standard deviation, summer 2019.

\begin{tabular}{cccccc}
\hline Lake & Month & Rotifera & Cladocera & Copepoda & Total \\
\hline & & \multicolumn{4}{c}{ Abundance, Thousand ind./m } \\
\hline \multirow{2}{*}{ Ali } & June & $0.60 \pm 0.20$ & $3.31 \pm 0.25$ & $0.05 \pm 0.03$ & $4.01 \pm 0.01$ \\
& August & $0.80 \pm 0.61$ & $4.60 \pm 2.62$ & $1.70 \pm 0.21$ & $7.30 \pm 2.61$ \\
Pervomaika & June & $0.36 \pm 0.16$ & $2.28 \pm 0.52$ & $0.24 \pm 0.22$ & $2.93 \pm 0.66$ \\
& August & $0.87 \pm 0.65$ & $13.75 \pm 7.48$ & $2.29 \pm 1.51$ & $16.22 \pm 8.12$ \\
Derevyannoe & June & $1.65 \pm 1.14$ & $5.71 \pm 2.21$ & $0.91 \pm 0.52$ & $7.77 \pm 2.68$ \\
& August & $4.69 \pm 2.36$ & $1.64 \pm 1.56$ & $6.49 \pm 5.03$ & $12.82 \pm 2.27$ \\
Altynkol & June & $0.56 \pm 0.43$ & $3.76 \pm 0.82$ & $3.09 \pm 1.87$ & $7.42 \pm 3.15$ \\
& August & $5.58 \pm 0.61$ & $5.86 \pm 1.66$ & $11.91 \pm 4.01$ & $23.37 \pm 1.73$ \\
Kosagash & June & $0.04 \pm 0.03$ & $0.33 \pm 0.32$ & $6.91 \pm 4.81$ & $7.30 \pm 4.52$ \\
& August & $0.05 \pm 0.03$ & $0.02 \pm 0.01$ & $0.18 \pm 0.05$ & $0.26 \pm 0.08$ \\
Malaya & June & $0.81 \pm 0.35$ & $2.88 \pm 0.81$ & $0.01 \pm 0.01$ & $3.72 \pm 1.11$ \\
Podkova & August & $0.01 \pm 0.01$ & $0.24 \pm 1.78$ & $0.01 \pm 0.01$ & $0.25 \pm 0.17$ \\
Bolshaya & June & $2.13 \pm 0.13$ & $3.97 \pm 3.56$ & $162.92 \pm$ & $169.0 \pm 15.61$ \\
Podkova & August & 0.0 & $0.01 \pm 0.01$ & $0.03 \pm 0.01$ & $0.05 \pm 0.03$ \\
Lower & June & $0.30 \pm 0.10$ & $0.70 \pm 0.50$ & $1.11 \pm 0.10$ & $2.10 \pm 0.41$ \\
Kolsay & August & $0.61 \pm 0.31$ & $1.30 \pm 0.61$ & $1.24 \pm 0.80$ & $3.12 \pm 1.11$ \\
Middle & June & $2.40 \pm 1.10$ & $0.31 \pm 0.11$ & $1.10 \pm 0.10$ & $3.80 \pm 1.61$ \\
Kolsay & August & $0.05 \pm 0.03$ & $1.60 \pm 0.80$ & $0.10 \pm 0.01$ & $1.81 \pm 1.01$ \\
\hline
\end{tabular}

Table 8. Zooplankton biomass in small lakes of South-East Kazakhstan, mean values with standard deviation, summer 2019.

\begin{tabular}{|c|c|c|c|c|c|}
\hline \multirow{2}{*}{ Lake } & \multirow{2}{*}{ Month } & Rotifera & Cladocera & Copepoda & Total \\
\hline & & \multicolumn{4}{|c|}{ Biomass, $\mathrm{mg} / \mathrm{m}^{3}$} \\
\hline \multirow{2}{*}{ Ali } & June & $1.08 \pm 0.69$ & $15.03 \pm 2.51$ & $0.40 \pm 0.36$ & $16.52 \pm 2.18$ \\
\hline & August & $0.78 \pm 0.38$ & $12.58 \pm 3.16$ & $12.53 \pm 5.51$ & $26.04 \pm 1.83$ \\
\hline \multirow[b]{2}{*}{ Pervomaika } & June & $0.36 \pm 1.67$ & $2.28 \pm 0.52$ & $0.24 \pm 0.22$ & $2.90 \pm 0.66$ \\
\hline & August & $0.10 \pm 0.07$ & $99.30 \pm 2.40$ & $37.81 \pm 27.20$ & $137.30 \pm 0.50$ \\
\hline \multirow{2}{*}{ Derevyannoe } & June & $10.40 \pm 0.01$ & $26.70 \pm 0.01$ & $3.80 \pm 2.50$ & $40.90 \pm 0.10$ \\
\hline & August & $56.70 \pm 28.60$ & $10.01 \pm 9.80$ & $14.09 \pm 9.10$ & $81.07 \pm 25.30$ \\
\hline \multirow{2}{*}{ Altynkol } & June & $3.50 \pm 2.80$ & $65.09 \pm 1.20$ & $38.81 \pm 19.20$ & $108.30 \pm 1.20$ \\
\hline & August & $5.51 \pm 0.60$ & $5.80 \pm 1.07$ & $11.09 \pm 4.01$ & $23.3 \pm 1.70$ \\
\hline \multirow{2}{*}{ Kosagash } & June & $0.30 \pm 0.20$ & $1.50 \pm 1.03$ & $74.33 \pm 1.30$ & $76,01 \pm 1,94$ \\
\hline & August & $0.19 \pm 0.20$ & $0.10 \pm 0.03$ & $0.21 \pm 0.07$ & $0.51 \pm 0.20$ \\
\hline Malaya & June & $2.80 \pm 1.20$ & $7.31 \pm 1.10$ & $0.21 \pm 0.16$ & $10.40 \pm 4.41$ \\
\hline Podkova & August & $0.11 \pm 0.05$ & $1.10 \pm 0.80$ & $0.11 \pm 0.01$ & $1.30 \pm 0.81$ \\
\hline Bolshaya & June & $9.40 \pm 1.33$ & $8.80 \pm 1.80$ & $330.70 \pm 1.50$ & $349.01 \pm 0.81$ \\
\hline Podkova & August & 0.0 & $0.03 \pm 0.01$ & $0.41 \pm 0.20$ & $0.51 \pm 0.21$ \\
\hline Lower & June & $2.50 \pm 1.10$ & $24.10 \pm 0.81$ & $31.67 \pm 1.70$ & $58.41 \pm 1.01$ \\
\hline Kolsay & August & $6.71 \pm 2.10$ & $61.71 \pm 1.07$ & $20.10 \pm 1.60$ & $88.56 \pm 1.09$ \\
\hline Middle & June & $49.01 \pm 1.10$ & $7.04 \pm 1.01$ & $32.01 \pm 0.16$ & $88.51 \pm 1.91$ \\
\hline Kolsay & August & $4.40 \pm 1.20$ & $51.10 \pm 6.05$ & $8.01 \pm 1.90$ & $63.50 \pm 5.31$ \\
\hline
\end{tabular}

Cladocerans dominated in abundance, whereas copepod species prevailed in biomass (Table 9). The composition of dominants remained relatively permanent during the summer in lakes Ali, Pervomaika, Malaya Podkova, and Bolshaya Podkova. Rotifers B. plicatilis, cladoceran $B$. longirostris and copepodite stages of cyclopoids Thermocyclops were dominant. The dominant species included A. priodonta, S. vetulus, D. (Daphnia) galeata, Diaphanosoma sp., T. taihokuensis, A. denticornis in other lakes. 
Table 9. Composition of dominant species in zooplankton of small lakes in South-East Kazakhstan, summer 2019.

\begin{tabular}{|c|c|c|c|c|c|}
\hline Lake & Month & Group & Species & Abundance, $\%$ & Biomass, $\%$ \\
\hline \multirow{4}{*}{ Ali } & June & Cladocera & B.(B) longirostris & 82.70 & 79.07 \\
\hline & \multirow{2}{*}{ August } & Cladocera & B.(B) longirostris & 57.21 & 42.37 \\
\hline & & Copepoda & Cyclopoida gen.sp. & 24.50 & 48.85 \\
\hline & June & Cladocera & B.(B) longirostris & 74.65 & 71.65 \\
\hline \multirow[t]{2}{*}{ Pervomaika } & \multirow{2}{*}{ August } & Cladocera & B.(B) longirostris & 78.91 & 37.67 \\
\hline & & Copepoda & Th. taihokuensis & 13.10 & 26.82 \\
\hline \multirow{4}{*}{ Derevyannoe } & \multirow{2}{*}{ June } & Cladocera & B.(B) longirostris & 73.01 & 65.07 \\
\hline & & Rotifera & A. priodonta & 10.20 & 23.47 \\
\hline & \multirow{2}{*}{ August } & Copepoda & Cyclopoida gen.sp. & 50.41 & 17.52 \\
\hline & & Rotifera & A. priodonta & 36.45 & 69.32 \\
\hline \multirow{6}{*}{ Altynkol } & \multirow{4}{*}{ June } & Copepoda & Th. crassus & 25.55 & 27.17 \\
\hline & & Cladocera & S. vetulus & 16.04 & 9.12 \\
\hline & & Copepoda & Cyclopoida gen.sp. & 16.24 & 8,70 \\
\hline & & Cladocera & Diaphanosoma sp. & 7.74 & 22.02 \\
\hline & \multirow{2}{*}{ August } & Copepoda & Cyclopoida gen.sp. & 48.74 & 49.41 \\
\hline & & Cladocera & B.(B) longirostris & 20.14 & 16.11 \\
\hline \multirow{3}{*}{ Kosagash } & \multirow{2}{*}{ June } & Copepoda & Cyclopoida gen.sp. & 67.14 & 32.10 \\
\hline & & Copepoda & Th. taihokuensis & 25.14 & 42.04 \\
\hline & August & Copepoda & Cyclopoida gen.sp. & 95.12 & 95.14 \\
\hline \multirow{3}{*}{ Malaya Podkova } & \multirow{2}{*}{ June } & Cladocera & B.(B) longirostris & 76.24 & 70.14 \\
\hline & & Rotifera & B. plicatilis & 13.17 & 24.34 \\
\hline & \multirow{2}{*}{$\begin{array}{l}\text { August } \\
\text { June }\end{array}$} & Cladocera & B.(B) longirostris & 92.27 & 88.41 \\
\hline Bolshaya & & Copepoda & Cyclopoida gen.sp. & 96.27 & 94.34 \\
\hline \multirow[t]{2}{*}{ Podkova } & August & Copepoda & Cyclopoida gen.sp. & 96.27 & 94.31 \\
\hline & \multirow{2}{*}{ June } & Copepoda & Cyclopoida gen.sp. & 44.57 & 23.94 \\
\hline \multirow{3}{*}{ Lower Kolsay } & & Cladocera & D. (Daphnia) galeata & 28.44 & 38.32 \\
\hline & \multirow{2}{*}{ August } & Cladocera & D. (Daphnia) galeata & 38.84 & 67.87 \\
\hline & & Copepoda & Cyclopoida gen.sp. & 34.62 & 16.27 \\
\hline \multirow{4}{*}{ Middle Kolsay } & \multirow{2}{*}{ June } & Rotifera & A. priodonta & 50.64 & 55.01 \\
\hline & & Copepoda & A. denticornis & 18.37 & 21.03 \\
\hline & \multirow{2}{*}{ August } & Cladocera & D. (Daphnia) galeata & 85.07 & 80.42 \\
\hline & & Copepoda & A. denticornis & 4.22 & 10.47 \\
\hline
\end{tabular}

The lowest Shannon diversity of zooplankton found in Bolshaya Podkova in August. The highest zooplankton diversity recorded in Ali and Altynkol lakes. It did not change significantly throughout the season (Table 10). In August zooplankton diversity in the lakes Malaya Podkova, and Middle Kolsay was lower than in June.

Table 10. Structural variables of zooplankton in small lakes in South-East Kazakhstan, mean values with standard deviation, summer 2019.

\begin{tabular}{|c|c|c|c|c|c|}
\hline Lake & Month & Species Number & Shannon $\mathrm{Ab}$ & Shannon Bi & $\begin{array}{c}\text { Average Individual } \\
\text { Mass of an } \\
\text { Organism, mg }\end{array}$ \\
\hline \multirow{2}{*}{ Ali } & June & 7 & $2.06 \pm 0.38$ & $2.32 \pm 0.01$ & $0.004 \pm 0.001$ \\
\hline & August & 12 & $2.07 \pm 0.02$ & $1.92 \pm 0.10$ & $0.004 \pm 0.001$ \\
\hline \multirow[b]{2}{*}{ Pervomaika } & June & 22 & $1.30 \pm 0.45$ & $1.26 \pm 0.50$ & $0.003 \pm 0.001$ \\
\hline & August & 13 & $1.24 \pm 0.40$ & $1.20 \pm 0.01$ & $0.007 \pm 0.002$ \\
\hline \multirow{2}{*}{ Derevyannoe } & June & 13 & $0.92 \pm 0.20$ & $0.84 \pm 0.32$ & $0.004 \pm 0.001$ \\
\hline & August & 10 & $0.84 \pm 0.50$ & $0.72 \pm 0.38$ & $0.007 \pm 0.002$ \\
\hline \multirow{2}{*}{ Altynkol } & June & 10 & $2.07 \pm 0.39$ & $2.32 \pm 0.04$ & $0.015 \pm 0.001$ \\
\hline & August & 15 & $2.07 \pm 0.02$ & $1.92 \pm 0.10$ & $0.005 \pm 0.001$ \\
\hline \multirow{2}{*}{ Kosagash } & June & 9 & $1.14 \pm 0.42$ & $1.12 \pm 0.04$ & $0.013 \pm 0.005$ \\
\hline & August & 10 & $1.52 \pm 0.16$ & $1.60 \pm 0.01$ & $0.003 \pm 0.001$ \\
\hline \multirow{2}{*}{ Malaya Podkova } & June & 15 & $1.16 \pm 0.12$ & $1.21 \pm 0.01$ & $0.002 \pm 0.001$ \\
\hline & August & 4 & $0.68 \pm 0.37$ & $0.83 \pm 0.56$ & $0.005 \pm 0.001$ \\
\hline
\end{tabular}


Table 10. Cont.

\begin{tabular}{cccccc}
\hline Lake & Month & Species Number & Shannon Ab & Shannon Bi & $\begin{array}{c}\text { Average Individual } \\
\text { Mass of an } \\
\text { Organism, mg }\end{array}$ \\
\hline \multirow{2}{*}{ Bolshaya Podkova } & June & 10 & $1.16 \pm 0.12$ & $0.67 \pm 0.34$ & $0.002 \pm 0.001$ \\
& August & 2 & $0.42 \pm 0.16$ & $0.49 \pm 0.00$ & $0.003 \pm 0.001$ \\
Lower Kolsay & June & 23 & $1.77 \pm 0.14$ & $1.59 \pm 0.14$ & $0.021 \pm 0.005$ \\
& August & 16 & $1.39 \pm 0.45$ & $1.08 \pm 0.03$ & $0.027 \pm 0.005$ \\
Middle Kolsay & June & 15 & $1.60 \pm 0.13$ & $1.58 \pm 0.14$ & $0.025 \pm 0.005$ \\
& August & 12 & $0.66 \pm 0.35$ & $0.66 \pm 0.35$ & $0.032 \pm 0.005$ \\
\hline
\end{tabular}

Zooplankton had a small size composition in Malaya Podkova, Bolshaya Podkova, Kosagash, Derevyannoe, Altynkol, Ali, Pervomayka. On the contrary, zooplankton of Middle and Lower Kolsay consisted of large-sized individuals.

\subsection{Redundancy Analysis, RDA}

There was an attempt to find the influence of environmental variables on the zooplankton structure of the surveyed lakes. According to the sum of all canonical values, $42 \%$ of the variation in the structure of zooplankton communities can be explained by the influence of environmental factors. The first RDA axis justifies $23.87 \%$ of the variation in zooplankton quantitative variables of surveyed lakes in summer 2019. TDS accounts for $19.3 \%$ of the total variation. Transparency and nitrate content explain $16.9 \%$ and $11.0 \%$ variation, respectively. Phytoplankton biomass clarified $6.0 \%$ of zooplankton abundance variation. As a result, the effects of the mentioned above environmental factors on zooplankton abundance were insignificant $(p=0.06)$. The main reason can be the distribution in small lakes species which adapted to changeable environmental conditions. However, despite the statistically insignificant relationship, we decided to consider each factor separately and identify the relationship between species of zooplankton and environmental variables.

According to the RDA biplot (Figures 5 and 6), three groups of factors are associated with certain lakes. The first group of factors included transparency and nitrate contents. The highest values of these variables were associated with the mountain Kolsay lakes. The second factor that has a significant impact on the zooplankton was TDS and phytoplankton biomass. Lakes Pervomaika, Derevyannoe, Kosagash, Malaya Podkova, and Bolshaya Podkova were connected with this factor. The third group of factors included temperature and phosphate concentration. This group of factors covered zooplankton communities of Kosagash, Malaya Podkova, Bolshaya Podkova, Ali, and Altynkol lakes.

Redundancy Analysis revealed zooplankton species that are most sensitive to changes in environmental factors (negative correlation) and species that prefers such conditions (positive correlation). Transparency, temperature, TDS, content of nitrates, phosphates, and phytoplankton biomass were the main controlling factors for the dynamics of quantitative variables of zooplankton.

A positive relationship was recorded between $D$. galeata and water transparency; rotifers A. brightwelli, K. quadrata, F. longiseta, Synchaeta sp., cladocerans D. longispina, D. longiremis, copepods E. serrulatus, A. denticornis, $C$. vicinus and the concentration of nitrates (Figure 5). A positive correlation was established between B. plicatilis, T. taihokuensis, and phytoplankton biomass. Rotifers Brachionus quadridentatus and cladocerans $A$. rectangula were positively influenced by TDS, while the rotifers B. hudsoni and cladocerans B. longirostris were affected by water temperature. Quantitative variables of $S$. vetulus and T. crassus positively correlated with the concentration of phosphates. 


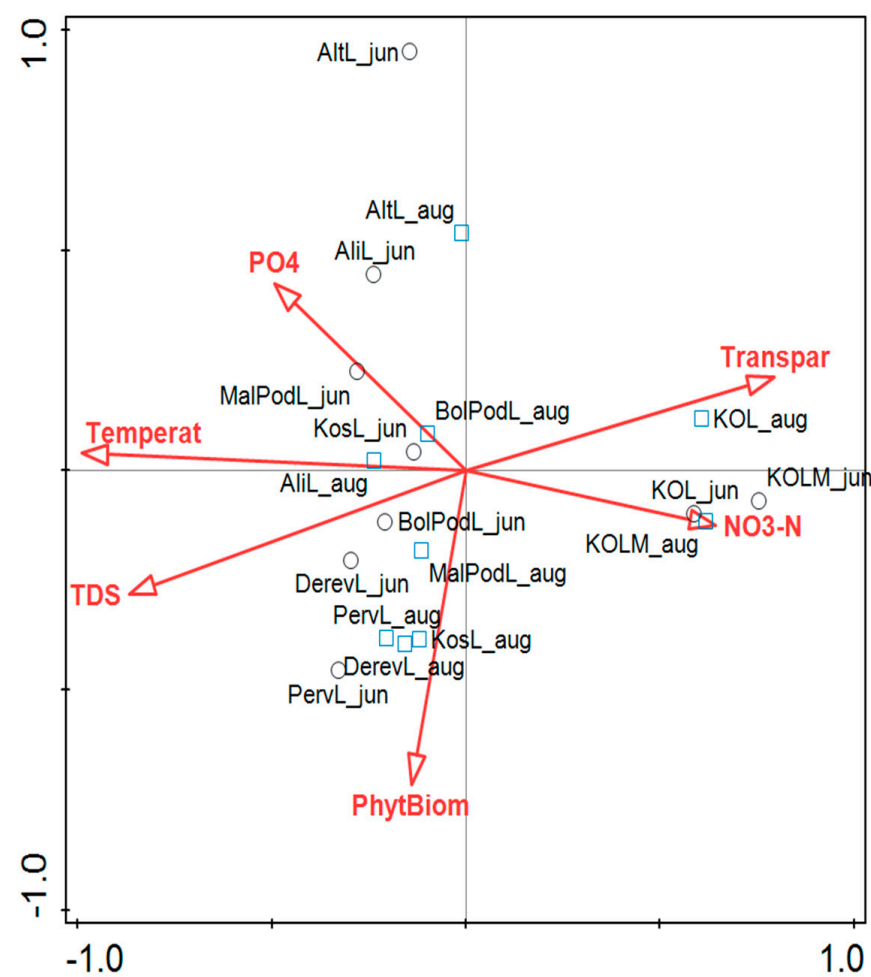

Figure 5. Redundancy Analysis, RDA results based on quantitative variables of zooplankton species. Circles-June, quadrangles-August, red arrows-environmental variables. Abbreviations used in the figure: AliL_Ali, PervL-Pervomaika, DerevL-Derevyannoe, AltL—Altynkol, KosL-Kosagash, MalPodL_Malaya Podkova, BolPodL_Bolshaya Podkova, KOL_Lower Kolsay, KOLM-Middle Kolsay.

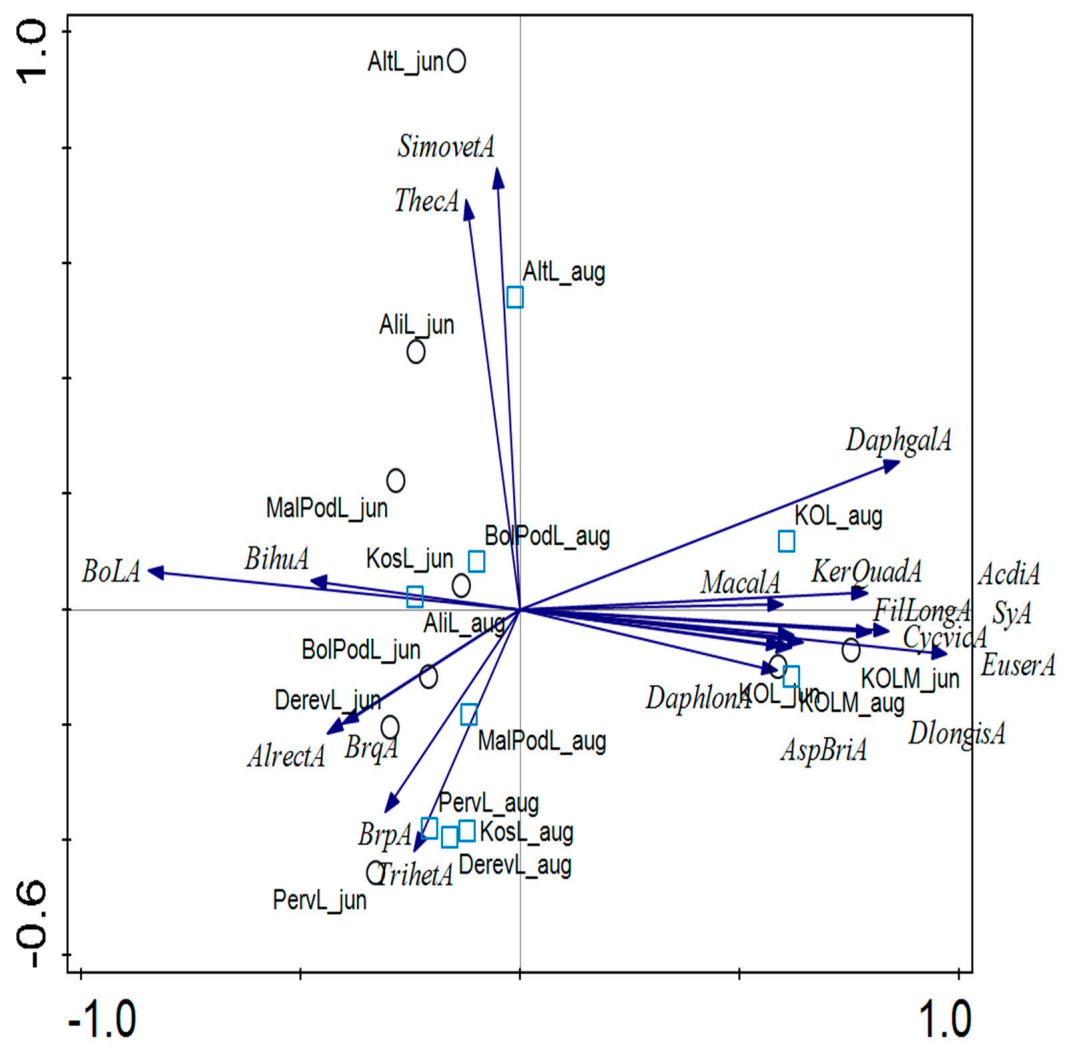

Figure 6. RDA correlation biplot of zooplankton and environmental variables. Circles-June, 
quadrangles-August, blue arrows-biological variables. Abbreviations used in the figure: AspBriAabundance of Asplanchna brightwelli, D.longisA—abundance of Daphnia longispina, D.lonA-abundance of D.longiremis, EuserA-abundance of Eucyclops serrulatus, CycvicA-abundance of Cyclops vicinus, SyA-abundance of Synchaeta, Fillong-abundance of Filinia longiseta, Acdi-abundance of Acantodiaptomus denticornis, MacalA-abundance of Macrocyclops albidus, KerquadA—abundance of Keratella quadrata, DaphgalA—abundance of D.galeata, ThecA-abundance of T. crassus, SimovetA—S. vetulus, BrpA-abundance of B. plicatilis, TrihetA-abundance of T. taihokuensis, BrqA—abundance of Brachionus quadridentatus, BrpA-abundance of Brachionus plicatilis, Alrect-abundance of Alona rectangula, BoLA—abundance of Bosmina longirostris, BihuA-abundance of Bipalpus hudsoni.

\section{Discussion}

\subsection{Chemical Variables}

According to the hydrochemical analysis results, mountain lakes belong to the category of ultra-freshwater bodies and the lakes at lower altitudes had freshwater [60]. The permanganate index of the water of mountain lakes and phosphates content were significantly lower than in the lakes at a lower altitude. One feeding source (river), absence of agricultural land use, and therefore low inputs from the surrounding land are reasons for the low permanganate index values and concentrations of $\mathrm{PO}_{4}$ in high mountain lakes. High mountain lakes are generally oligotrophic [61].

With a relatively low content of nitrites, the concentrations of nitrates and ammonium were elevated in all lakes in comparison with some small lakes in other arid regions [62]. In general, during summer, chemical parameters in the surveyed lakes varied significantly, which also noted for small lakes in other regions [1,3].

\subsection{Phytoplankton}

\subsubsection{Species Composition and Quantitative Variables}

In summer of 2019, the phytoplankton of the surveyed lakes was represented by 74 taxa. A similar number of taxa were found in the phytoplankton of shallow water bodies in other regions of Kazakhstan (Northern Kazakhstan), Turkey, and Poland [63-65]. According to the network analysis results, phytoplankton species composition in the lakes at higher altitude differed from the composition of phytoplankton species in the lakes at lower altitudes. the species composition of phytoplankton in the lakes at lower altitudes changed significantly during one summer season. Such a significant difference in phytoplankton species composition in one lake during one season is due to the variability of hydrochemical parameters in the surveyed lakes [1].

The abundance of phytoplankton in the surveyed lakes varied significantly, from $8.5 \times 10^{7}$ to $2.71667 \times 10^{9}$ cells $/ \mathrm{m}^{3}$ with a biomass from 0.4 to $15.81 \mathrm{~g} / \mathrm{m}^{3}$. The minimum values of the abundance and biomass of communities were recorded in Lake Altynkol (abundance $8.5 \times 10^{7}$ cells $/ \mathrm{m}^{3}$, biomass $0.52 \mathrm{~g} / \mathrm{m}^{3}$ ). These results were close to the values established for the most polluted water bodies of Argentina (abundance $6 \times 10^{6}$ to $8.30 \times 10^{6}$ cells $/ \mathrm{m}^{3}$, biomass from $0.02 \mathrm{~g} / \mathrm{m}^{3}$ to $0.3 \mathrm{~g} / \mathrm{m}^{3}$ ) and Turkey (biomass $\left.0.001 \mathrm{~g} / \mathrm{m}^{3}-0.05 \mathrm{~g} / \mathrm{m}^{3}\right)[64,66]$. The highest abundance of phytoplankton established in some lakes at a lower altitude was comparable to the quantitative variables of microalgae in wastewater reservoirs in South-East Kazakhstan (from $5.8917 \times 10^{9}$ to $6.2876 \times 10^{9}$ cells $/ \mathrm{m}^{3}$ and $4.77-5.62 \mathrm{~g} / \mathrm{m}^{3}$ ) [67].

The increased quantitative variables of phytoplankton in the lakes (especially in Bolshaya Podkova, Kosagash, Lower Kolsay) from June to August connected with the fluctuations of nutrients concentrations. It is known that an increase in the amount of organic matter stimulates the development of microalgae [68]. Cyanobacteria dominated in abundance almost all surveyed water bodies, except for the Middle Kolsay, Ali, and Pervomayka. Cyanobacteria prevail mainly in water bodies with organic pollution $[64,68]$. The variability of hydrochemical parameters, especially phosphate concentration increase, led to cyanobacteria dominance in water bodies [68]. In addition to Cyanobacteria, the basis of quantitative variables of algal communities was formed by Diatoms, Miozoa, and 
Chlorophyta. Some of them, for example, Ceratium hirundinella, like cyanobacteria, can cause water bodies to bloom [69-71]. Ceratium hirundinella was widespread in the surveyed lakes at a lower altitude.

\subsubsection{Structural Variables}

Shannon phytolankton diversity varied from 0.46 to 3.69 , but more often, it was at a moderate level-from 1.34 to 2.70 in the surveyed lakes. Similar Shannon diversities were found in water bodies of Turkey and Denmark with organic pollution [64,72]. Shannon's diversity index was very low (around 0.44-0.70) in wastewater reservoirs of South-East Kazakhstan, with the extreme level of pollution [67].

In the lakes located at a lower altitude, phytoplankton consisted of small-size species. Generally, small-size species composition is typical of anthropogenically modified water bodies [67]. It is known that a high content of nutrients leads to the dominance of small-size species of Cyanobacteria [68]. The increased values of algae cell volume were recorded in Pervomayka, Derevyannoe, Middle Kolsay, Lower Kolsay, where large algal species of Bacillariophyta, Chlorophyta, and Miozoa dominated (Table 4).

\subsection{Zooplankton}

\subsubsection{Species Composition and Quantitative Variables}

In summer 2019, 58 taxa were identified in zooplankton of South-East Kazakhstan small lakes. The low species richness is characteristic for zooplankton in small lakes of different regions [35-37,73,74], including mountain lakes [75-77].

According to the analysis of literature data and the obtained results, some planktonic invertebrates which are characteristic of small water bodies can be distinguished. Rotifers Asplanchna priodonta, Keratella cochlearis, K. quadrata, Filinia longiseta, F. terminalis, Daphnia (Daphnia) longispina [75-77], copepods of the genus Eudiaptomus and Acantodiaptomus [33] noted in our study were typical species of zooplankton in mountain cold-water lakes. Some plankton invertebrates are typical of plain shallow water bodies. These include rotifers Asplanchna priodonta, Bipalpus hudsoni, Brachionus angularis, cladocerans Alona rectangula, Chydorus sphaericus, Bosmina longirostris, and cyclops Thermocyclops crassus [78]. The same species of planktonic invertebrates were also recorded in the surveyed small water bodies. The listed complex of widespread species is also typical of water bodies under the influence of planktivorous fish [79-81]. In the absence of a press of planktivorous fish and in water bodies with macrophytes, crustaceans D.pulex, Ceriodaphnia quadrangula were typical species in zooplankton in Kazakstan [36] and other regions [82,83].

According to the network analysis results, zooplankton species composition in the surveyed lakes at a lower altitude changed significantly from June to August compared to lakes at higher altitudes. Unstable species composition of planktonic invertebrates in the surveyed shallow lakes under the variability of environmental factors was noted for the shallow lakes of other regions [1].

The quantitative variables of zooplankton in the surveyed freshwater small lakes varied by two orders of magnitude, but in general, they were at a lower level (Tables 5 and 6 ) than in the lakes with high TDS (up to 175.0 thousand ind. $/ \mathrm{m}^{3}$ and $2.8 \mathrm{~g} / \mathrm{m}^{3}$ ) [36]. The increase of zooplankton abundance in the gradient of TDS can be due to a decrease in the pressure of planktivorous fish, which are sensitive to hydrochemical parameters [79-81]. The data we identified on the quantitative variables of zooplankton differed from the data established for highly polluted (organic and toxic) water bodies of South-East Kazakhstan (with zooplankton abundance up to 657.0 thousand ind. $/ \mathrm{m}^{3}$ with biomass of up to $17 \mathrm{~g} / \mathrm{m}^{3}$ ) [26].

Along with TDS, water temperature is the main factor forming the species composition and abundance of zooplankton. In the mountainous cold-water Kolsay lakes, the quantitative variables of zooplankton were lower compared to some warm-water plain lakes in the region (Tables 7 and 8), and ten times less compared to mountain lakes in Italy [75], Ethiopia [76], and America [77]. 


\subsubsection{Structural Variables}

Zooplankton consisted of small-sized species in the lakes at a lower altitude (Table 9). The low value of individual mass was due to the dominance of small cyclops Thermocyclops crassus and T. taihokuensis. Among the dominants most often were the small species Bosmina (Bosmina) longirostris, which is also typical for zooplankton of small water bodies in other regions [78] under the influence of planktivorous fish [79-81].

The large-sized species of zooplankton, rotifers Asplanchna priodonta and cladocerans D. (Daphnia) galeata dominated in Kolsay mountain lakes (Table 9). The main reason for the differences in the size variables of zooplankton in the lakes at a higher altitude and in lakes at a lower altitude can be differences in temperature, the degree of macrophyte cover, and the content of nutrients. In general, the increasing role of small zooplankton species occurs under conditions of development of cyanobacteria, which suppress the large-sized species as Daphnia [84,85]. According to the obtained data cyanobacteria dominated almost all the surveyed water bodies, except for the Middle Kolsay, Ali, and Pervomayka.

Shannon diversity of zooplankton varied significantly from 0.42 to 2.30 (Table 9). The diversity of zooplankton communities in mountain lakes was lower than in lakes at a lower altitude. The similar values of Shannon's diversity index were noted in some moderately polluted water bodies of Kazakhstan and other regions [26,37,85,86].

4.3.3. The Influence of External Factors on the Quantitative Variables and Structure of Zooplankton Communities

Multivariate analysis (Figures 4 and 5) made it possible to identify the main factors influencing zooplankton structure in the surveyed small lakes. The first group of factors included water transparency and nitrate content. Zooplankton communities of the Kolsay mountain lakes were associated with these group of factors. Crustaceans Daphnia (Daphnia) galeata, D. longispina, D. longiremis, Eucyclops serrulatus, Acanthodiaptomus denticornis, Cyclops vicinus dominated in zooplankton communities of the Kolsay mountain lakes. It is known that large-sized cladocerans (for example, D. galeata) contribute to the increasing of water transparency [87] due to more efficient filtration of bacteria, ultraand nanoplankton [88]. With an increasing concentration of nitrates, abundance of some representatives of zooplankton, mainly cyclops and species of Diaptomidae, increased. This feature is characteristic of the eutrophic water bodies, where primarily colonial forms of algae develop, which are consumed by cyclops and species of Diaptomidae [87,89].

The second group of environmental factors included temperature and phosphates. Zooplankton communities of lakes Kosagash, Malaya Podkova, Bolshaya Podkova Ali, and Altynkol were related to this group. According to the results of the RDA analysis small-sized B. longirostris dominated in zooplankton of studied lakes, with a water temperature gradient from $22^{\circ} \mathrm{C}$ to $27^{\circ} \mathrm{C}$, which was also noted in lakes of other regions [90]. However, higher water temperatures from $27^{\circ} \mathrm{C}$ to $32{ }^{\circ} \mathrm{C}$ cause high mortality of these cladocerans [91].

The increased level of phosphates in freshwater ecosystems contributes to the massive development of cyanobacteria [68,92], which was noted for the lakes listed above. According to the results of the RDA analysis, Bipalpus hudsoni, Simocephalus vetulus, Thermocyclops sp. resistant to high contents of phosphate. In many studies, the maximum abundance and biomass of Thermocyclops sp. were recorded in eutrophic water bodies, where the quantitative variables of Cyanobacteria are high [68]. Among potentially harmful cyanobacteria species, there were Gomphosphaeria aponia Kützing, Merismopedia punctata Meyen, Microcystis aeruginosa f. flos-aquae (Wittrock) Elenkin in the surveyed lakes [84,93]. Some species of cyclops Thermocyclops sp. can use cyanobacteria as food [94,95]. At the same time, cyanobacteria negatively affect the species of the genus Daphnia due to clogging of the digestive system, the formation of large colonies with mucus, the production of toxins and the low nutritional quality of cells $[96,97]$.

The third group of factors included TDS and phytoplankton biomass as the food base for planktonic invertebrates. This group united lakes Bolshaya Podkova, Malaya Podkova, 
Derevyannoe, Pervomayka, Kosagash and Ali. According to the RDA analysis, the total content of dissolved solids had a weak effect only on the abundance of two species-rotifera Brachionus quadrudentatus and cladocera Alona rectangula. This is primarily due to the small TDS gradient in the surveyed lakes and the resistance of the listed species to TDS up to $1000 \mathrm{mg} / \mathrm{dm}^{3}$ [98,99]. Moderately strong positive relationships were recorded between rotifera B. plicatilis and cyclop Thermocyclops taihokuensis. It is known that rotifers have a short development time, a high filtration rate, and consuming food resources continuously, therefore reach a high density quickly [100]. These characteristics make them successful in controlling the high density of phytoplankton.

4.3.4. Indicator Role of Zooplankton in Assessing the Water Quality of Water Bodies with Organic Pollution

The species composition, abundance, biomass, Shannon's diversity index, and size structure of aquatic communities are usually used to assess organic pollution of water bodies [25]. The abundance of large-sized zooplankton species, which are the best filter feeders (for example, species of the genus Daphnia), sharply decrease in eutrophic conditions. The dominance of small-sized species (rotifers, small cladocerans, cyclops) decreases the size structure [101]. The dominance of a few species is responsible for the low values of the Shannon's diversity [25].

Changes in zooplankton structure with increasing organic pollution are associated with corresponding changes in phytoplankton communities as a food base for zooplankton [68]. Colonial forms of algae such as Cyanobacteria and large Miozoa species begin to dominate in the phytoplankton community $[68,102]$. Due to the dominance of large-sized Miozoa species, which are not consumed by small-sized zooplankton species, phytoplankton biomass can reach a high level.

The dominance of Cyanobacteria and Miozoa (Gomphosphaeria aponia, Merismopedia punctata, Microcystis flosaquae and Ceratium hirundinella) in phytoplankton indicated organic pollution of lakes at a lower altitude. This conclusion confirmed by the prevalence of rotifers and small cyclops Thermocyclops sp. in zooplankton. Rotifers are indicators of eutrophication [103-105]. The dominance of rotifers in the community is a signal of an increase in nutrients concentration [106]. It was evidenced with the established positive relationship between phytoplankton biomass and the abundance of the rotifera Brachionus plicatilis. B. plicatilis dominates mainly in the zooplankton of eutrophic water bodies [107, 108], however, some toxic microalgae species repress the development of this species [109]. The positive relationship between phosphate concentration and quantitative variables of Thermocyclops sp. confirms its indicator role in assessing the level of organic pollution of aquatic ecosystems. It can be assumed that the pollution of the surveyed lakes at a lower altitude occurs mainly due to the anthropogenic modifications of the surrounding land areas.

The positive relationship between nitrates and the quantitative variables of rotifers Asplanchna brightwelli, Keratella quadrata, Filinia longiseta, Synchaeta sp., crustaceans Daphnia (Daphnia) longispina, Eucyclops serrulatus, Acanthodiaptomus denticornis, Cylops vicinus evidenced the indicator role of these species in assessing organic pollution of the surveyed lakes. Keratella quadrata reached a high abundance under the conditions of a high recreational load of small lakes in Central Kazakhstan [110]. The literature review shows a decrease abundance of the species genus Daphnia with increasing organic pollution and cyanobacterial bloom [111]. Cyanobacteria species clogs filter apparatus of Daphnia [87]. However, D. longispina is an exception since it can destroy cyanobacteria trichomes [112]. Cyclops and diaptomus are also capable of consuming colonial forms of algae [87]. The pollution of the mountain lakes Middle and Lower Kolsay is probably related to increased recreational load.

Thus, the obtained results on the hydrochemical and biological variables of the mountain and lakes at a lower altitude in South-East Kazakhstan indicated organic pollution. The level of organic pollution is at an increased level in Kosagash, Bolshaya and Malaya Podkova lakes. The level of organic pollution is at a relatively low level in the rest of the 
lakes at a lower altitude. The Lower Kolsay is prone to organic pollution caused by an increase in the recreational load [33]. The Middle Kolsay is the cleanest lake due to its remoteness from sources of anthropogenic pollution.

\section{Conclusions}

In the summer of 2019, 58 taxa were identified in the zooplankton of small lakes in South-East Kazakhstan. Widespread zooplankton species in shallow lakes located at low altitudes were Asplanchna priodonta, Bosmina (Bosmina) longirostris, and Thermocyclops crassus. In cold-water mountain lakes, A. priodonta, cladocerans Daphnia (Daphnia) galeata and copepods Acanthodiaptomus denticornis, Cylops vicinus were most common. The small species Brachionus plicatilis, Bosmina (Bosmina) longirostris, and copepodite and nauplii of $T$. crassus dominated in the community of lakes at low altitudes. In the zooplankton communities of mountains lakes, Acanthodiaptomus denticornis and copepodite and nauplii of Cyclops vicinus prevailed. Species composition, abundance, and biomass of zooplankton in the surveyed lakes have been changed significantly during one summer season.

Multivariate analysis of RDA showed that the main factors influencing the structure of zooplankton communities in small lakes were temperature, TDS, nitrates, phosphates, and the composition and biomass of planktonic algae. The hydrochemical and biological data of the mountain and plain lakes in South-East Kazakhstan indicated the presence of varying degrees of organic pollution. Our results once again confirmed the applicability of structural variables of zooplankton in assessing water quality.

Author Contributions: Introduction, methodology, writing, M.A. editing, E.K.; investigation, Z.M.; data curation, funding acquisition, K.I. and S.A. All authors have read and agreed to the published version of the manuscript.

Funding: This research fully funded by the Ministry of Ecology, Geology and Natural Resources of the Republic of Kazakhstan (Natural Resources, Grant No. BP10264205). The work partly was carried out under project No. AP08855655, Institute of Zoology, the Committee of Science, Ministry of Education and Science, Republic of Kazakhstan "Assessment of the ecological state of wastewater reservoirs of the system of the Right-Bank Sorbulak Canal for the development of the scientific basis for wastewater disposal."

Institutional Review Board Statement: Not applicable.

Informed Consent Statement: Not applicable.

Data Availability Statement: No new data were created or analyzed in this study. Data sharing is not applicable to this article.

Acknowledgments: The authors would like to express deep gratitude and appreciation to Jaroslav Vrba from University of South Bohemia, Michal Šorf at Mendel University in Brno and Sophia Barinova at University of Haifa for helping to apply Multivariate statistical tools to the current research.

Conflicts of Interest: The authors declare no conflict of interest.

\section{References}

1. Meerhoff, M.; Jeppesen, E. Shallow lakes and ponds. In Encyclopedia of Inland Waters Edition; Pergamon Press: Oxford, UK, 2009; pp. 645-655.

2. Biggs, J.; Fumetti, S.; Kelly-Quinn, M. The Importance of Small Water Bodies: Insights from Research. Hydrobiologia 2017, 793, 1-2.

3. Scheffer, M. Alternative Attractors of Shallow Lakes. Sci. World. 2001, 1, 254-263. [CrossRef]

4. Tranvik, L.J.; Downing, J.B.; Cotner, S.A.; Loiselle, R.G.; Striegl, T.J.; Ballatore, P.J.; Dillon, K.; Finlay, K.; FortinO, L.B.; Knoll, P.; et al. Lakes and impoundments as regulators of carbon cycling and climate. Limnol. Oceanogr. 2009, 54, 2298-2314. [CrossRef]

5. Spoljar, M.; Drazina, T.; Sargac, J.; Kralj Borojevic, K.; Zutinic, P. Submerged macrophytes as a habitat for zooplankton development in two reservoirs of a flow-through system (Papuk Nature Park, Croatia). Intern. J. Limnol. 2012, 48, 161-175. [CrossRef]

6. Choi, J.Y.; Jeong, K.S.; La, G.H.; Joo, G.J. Effect of removal of free-floating macrophytes on zooplankton habitat in shallow wetland. Knowl. Manag. Aquat. Ecosyst. 2014, 414, 11-22. [CrossRef]

7. Feeley, H.B.; Kelly-Quinn, M. An evaluation of local and regional diversity of benthic macroinvertebrate communities in two small regions of Ireland and their potentialas localised refugia for certain taxonomic groups. Biol. Environ. Proc. R. Ir. Acad. 2012, 112, 43-54. [CrossRef] 
8. Diéguez, M.C.; Gilbert, J.J. Daphnia-rotifer interactions in Patagonian communities. Hydrobiologia 2010, 662, 189-195. [CrossRef]

9. Clarke, A.; Mac Nally, R.; Bond, N.; Lake, P.S. Macroinvertebrate diversity in headwater streams: A review. Freshw. Biol. 2008, 53, 1707-1721. [CrossRef]

10. Brysiewicz, A.; Sługocki, Ł.; Wesołowski, P.; Czerniawski, R. Zooplankton community structure in small ponds in relation to fish community and environmental factors. Appl. Ecol. Environ. Res. 2017, 15, 929-941. [CrossRef]

11. Callanan, M.; Baars, J.R.; Kelly-Quinn, M. Macroinvertebrate communities of Irish headwater streams: Contribution to catchment biodiversity. Biol. Environ. Proc. R. Ir. Acad. 2014, 114, 143-162. [CrossRef]

12. Williams, P.; Whitfield, M.; Biggs, J.; Bray, S.; Fox, G.; Nicolet, P.; Sear, D. Comparative biodiversity of rivers, streams, ditches, shallow lakes and ponds in an agricultural landscape in Southern England. Biol. Conserv. 2004, 115, 329-341. [CrossRef]

13. Krupa, E.G.; Dobrokhotova, O.V.; Stuge, T.S. Fauna Calanoida (Crustacea, Copepoda) of Kazakhstan and Adjacent Territories; EtalonPrint: Almaty, Kazakhstan, 2016; p. 208, ISBN 978-601-80265-8-4. (In Russian)

14. Silva, W.M.; Matsumura-tundisi, T. Taxonomy, ecology, and geographical distribution of the species of the genus Thermocyclops Kiefer, 1927 (Copepoda, Cyclopoida) IN SÃo Paulo state, Brazil, with description of a new species. Braz. J. Biol. 2005, 65, 521-531. [CrossRef] [PubMed]

15. De Bie, T.; Declerck, S.; Martens, K.; DeMeester, L.L. A comparative analysis of cladoceran communities from different water body types: Patterns in community composition and diversity. Hydrobiologia 2008, 597, 19-27. [CrossRef]

16. Taizhanov, E.B.; Kuzmetov, A.R.; Krupa, E.G.; Stuge, T.S.; Mirabdullayev, I.M. Fauna of Cyclops of Kazakhstan (Crustacea, Copepoda, Cyclopidae). In Proceedings of the Materials of the International Scientific Practical Conference (Urgent Problems of Ecology and Nature Use in Kazakhstan and Adjacent Territories 2007), Almaty, Kazakhstan, 12-15 November 2007; pp. 400-402. (In Russian).

17. Stepanova, L.A. Arctodiaptomus (Arctodiaptomus) naurzumensis n. sp. (Copepoda, Calanoida) from Kazakhstan. Hydrobiologia 1994, 288, 129-134. [CrossRef]

18. Krupa, E.G.; Stuge, T.S. New species of the genus Gigantodiaptomus (Copepoda, Calanoida) from Northeastern Kazakhstan. News NAS RK Ser. Biol. Med. 2007, 1, 18-22. (In Russian)

19. Bolpagni, R.; Bartoli, M.; Viaroli, P. Species and functional plant diversity in a heavily impacted riverscape: Implications for threatened hydro-hygrophilous flora conservation. Limnologica 2013, 43, 230-238. [CrossRef]

20. Hunter, M.L.; Acuña, V.; Bauer, D.M.; Bell, K.P.; Calhoun, A.J.K.; Felipe-Lucia, M.R.; Fitzsimons, J.A.; González, E.; Kinnison, M.; Lindenmayer, D.; et al. Conserving small natural features with large ecological roles: A synthetic overview. Biol. Conserv. 2017, 211, 88-95. [CrossRef]

21. Maynou, X.; Martín, R.; Aranda, D. The role of small secondary biotopes in a highly fragmented landscape as habitat and connectivity providers for dragonflies (Insecta: Odonata). J. Insect Conserv. 2017, 21, 517-530. [CrossRef]

22. Kaufman, Z.S. The Origin of Freshwater Biota; The Karelian Science Center of RAS: Petrozavodsk, Russia, 2005 ; pp. 150-220.

23. Krupa, E.G. Assessment of Changes in the Structure of Zooplankton Communities to Infer Water Quality of the Caspian Sea. Diversity 2019, 11, 122. [CrossRef]

24. Scheffer, M.; Geest, G.J.; Zimmer, K.; Jeppesen, E.; Sondergaard, M.; Butler, M.G.; Hanson, M.A.; Declerck, S.; DeMeester, L. Small habitat size and isolation can promote species richness: Second-order effects on biodiversity in shallow lakes and ponds. Oikos 2006, 112, 227-231. [CrossRef]

25. Andronikova, I.N. Structural and Functional Organization of Zooplankton of Lake Ecosystems of Various Trophic Types; Nauka: St. Petersburg, Russia, 1996; p. 189. (In Russian)

26. Krupa, E.; Barinova, S.; Romanova, S.; Aubakirova, M.; Ainabaeva, N. Planktonic Invertebrates in the Assessment of Long-Term Change in Water Quality of the Sorbulak Wastewater Disposal System (Kazakhstan). Water 2020, 12, 3409. [CrossRef]

27. Ismail, A.H.; Adnan, A.A. Zooplankton Composition and Abundance as Indicators of Eutrophication in Two Small Man-made Lakes. Trop. Life Sci. Res. 2016, 27, 31-38. [CrossRef] [PubMed]

28. Søndergaard, M.; Jeppesen, E.; Jensen, J.P. Pond or lake: Does it make any difference? Arch. Hydrobiol. 2005, 162, 143-165. [CrossRef]

29. Mirabdullayev, I.M.; Sharapova, L.I.; Stuge, T.S.; Kuzmetov, A.R. New records of Microcyclops afghanicus Lindberg, 1948 from Kazakhstan, Central Asia (Copepoda, Cyclopoida). Crustaceana 1997, 70, 849-854.

30. Krupa, E.G.; Barinova, S.S.; Assylbekova, S.Z.; Isbekov, K.B. Structural Indicators of Zooplankton of The Shardara Reservoir (Kazakhstan) And the Main Influencing Factors. Turk. J. Fish. Aquat. Sci. 2018, 18, 659-669. [CrossRef]

31. Barinova, S.; Krupa, E.; Tsoy, V.; Ponamareva, L. The Application of phytoplankton in ecological assessment of the Balkhash Lake (Kazakhstan). Appl. Ecol. Environ. Res. 2018, 16, 2089-2111. [CrossRef]

32. Krupa, E.G.; Barinova, S.S.; Romanova, S.M. Ecological Mapping in Assessing the Impact of Environmental Factors on the Aquatic Ecosystem of the Arys River Basin, South Kazakhstan. Diversity 2019, 11, 239. [CrossRef]

33. Krupa, E.G.; Barinova, S.S.; Romanova, S.M. Zooplankton size structure in the Kolsay mountain lakes and Its Relationships with Environmental Factors. Water Resour. 2019, 46, 403-404. [CrossRef]

34. Krupa, E.G.; Grishaeva, O. Impact of water salinity on long-term dynamics and spatial distribution of benthic invertebrates in the Small Aral Sea. Int. J. Oceanogr. Hydrobiol. 2019, 48, 355-367. [CrossRef]

35. Krupa, E.G. Species composition and seasonal dynamics of the abundance and biomass of lower crustaceans in the pond of the Central Park of Culture and Rest of Almaty. News NAS RK Ser. Biol. Med. 1998, 3, 72-75. (In Russian) 
36. Troshina, T.T. Biodiversity and structural characteristics of summer zooplankton in Almaty region (July and August 2010, 2012). News NAS RK Ser. Biol. Med. 2013, 3, 13-19. (In Russian)

37. Aubakirova, M.O. About zooplankton of small water bodies of Almaty city and Almaty region. In Proceedings of the Materials of the International Conference of Students and Young Scientists (Farabi alemi 2019), Almaty, Kazakhstan, 9-10 April 2019; pp. 8-9. (In Russian).

38. Uteshev, A.S. (Ed.) The Climate of Kazakhstan; Hydrometeorological Publishing House Leningrad: Leningrad, Russia, 1959; p. 360. (In Russian)

39. Cherednichenko, A.; Cherednichenko, V. Tropopause and Maximum Wind Over the Territory of Kazakhstan; AlFarabi Kazakh National University Litagent: Almaty, Kazakhstan, 2017; p. 515. (In Russian)

40. Abakumov, V.A. (Ed.) Guide on Methods for Hydrobiological Analysis of Surface Waters and Bottom Sediments; Gidrometeoizdat: Leningrad, Russia, 1983; p. 239. (In Russian)

41. Semenov, A.D. (Ed.) Guideline for Chemical Analysis of Surface Water; Gidrometeoizdat: Leningrad, Russia, 1977; p. 541. (In Russian)

42. Fomin, G.S. Water. Control of Chemical, Bacterial and Radiation Safety According to International Standards; NGO "Alternative": Moscow, Russia, 2000; p. 618. (In Russian)

43. Kiselev, I.A. Research methods of plankton. In Life of the Fresh Water of the USSR; Pavlovsky, E.N., Zhadin, V.I., Eds.; Academy of Sciences: Moscow, Russia; Leningrad, Russia, 1956; pp. 188-253. (In Russian)

44. Hollerbach, M.M.; Kosinskaya, E.K.; Polyansky, V.I. Determinant for Freshwater Algae of the USSR. The Blue-Green Algae; Soviet Science: Moscow, Russia, 1953; p. 654. (In Russian)

45. Matvienko, A.M. Determinant for Freshwater Algae of the USSR. Golden Algae; Soviet Science: Moscow, Russia, 1954; p. 189. (In Russian)

46. Moshkova, N.A.; Hollerbah, M.M. Determinant for Freshwater Algae of the USSR. Green Algae. Class Ulotrichous. Order Ulotrichous; Soviet Science: Moscow, Russia, 1986; p. 361. (In Russian)

47. Palamar-Mordvintseva, G.M. Determinant to Freshwater Algae of the USSR. Green Algae. Class Conjugates. Order Desmid; Soviet Science: Moscow, Russia, 1982; p. 621. (In Russian)

48. Popova, T.G. Determinant to Freshwater algae of the USSR. Euglena algae; Soviet Science: Moscow, Russia, 1955; p. 213. (In Russian)

49. Zabelina, M.M.; Kiselev, I.A.; ProshkinaLavrenko, A.I.; Sheshukova, V.S. Determinant for Freshwater Algae USSR. Diatoms; Soviet Science: Moscow, Russia, 1951; p. 622. (In Russian)

50. Kutikova, L.A. Rotifers of the Fauna of the USSR.; Science: Leningrad, Russia, 1964; p. 744. (In Russian)

51. Orlova-Bienkowskaja, M.Y. Cladocera: Anomopoda. Daphniidae: Genus Simocephalus; Backhuys Publishers: Leiden, The Netherlands, 2001; p. 130.

52. Manuilova, E.F. Cladocerans of the Fauna of the USSR; Nauka: Moscow, Russia, 1964; p. 328. (In Russian)

53. Rylov, V.M. Fauna of the USSR. Crustaceans. Freshwater Cyclopoida; Nauka: Moscow, Russia, 1948; p. 312. (In Russian)

54. Magurran, E. Ecological Diversity and Its Measurement; Mir: Moscow, Russia, 1998; p. 184, ISBN 5-03-002404-2. (In Russian)

55. Shitikov, V.K.; Rosenberg, G.S.; Zinchenko, T.D. Quantitative Hydroecology: Methods of Systemic Identification; Institute of Ecology of the Volga Basin of the Russian Academy of Sciences: Togliatti, Russia, 2003; p. 463, ISBN 5-93424-109-5. (In Russian)

56. Clarke, K.R.; Gorley, R.N. PRIMER v5: User Manual/Tutorial; PRIMER-E Ltd. Press: Plymouth, UK, 2001.

57. McAleece, N.; Lambshead, J.; Patterson, G.; Gage, J. BioDiversity Pro, Version 2; The Natural History Museum, London and The Scottish Association of Marine Science: Oban, UK, 1997.

58. Love, J.; Selker, R.; Marsman, M.; Jamil, T.; Dropmann, D.; Verhagen, A.J.; Ly, A.; Gronau, Q.F.; Smira, M.; Epskamp, S.; et al. JASP: Graphical statistical software for common statistical designs. J. Stat. Softw. 2019, 88, 1-17. [CrossRef]

59. Šmilauer, P.; Lepš, J. Multivariate Analysis of Ecological Data Using Canoco 5; Cambridge University Press: Cambridge, UK, 2014; p. 527.

60. Guseva, T.V. Hydrochemical Variables of the State of the Environment; Social and Environmental Union: Moscow, Russia, $2002 ;$ p. 148. (In Russian)

61. Catalan, J.; Camarero, L.; Felip, M.; Pla, S.; Ventura, M.; Buchaca, T.; De Quijano, D.D. High mountain lakes: Extreme habitats and witnesses of environmental changes. Limnetica 2006, 25, 551-584.

62. Moiseenko, T.I.; Skjelkvåle, A.D.; Gashkina, B.L.; Shalabodov, N.A.; Khoroshavin, V.Y. Water chemistry in small lakes along a transect from boreal to arid ecoregions in European Russia: Effects of air pollution and climate change. Appl. Geochem. 2013, 28, 69-79. [CrossRef]

63. Toleuzhanova, A.T. Phytoplankton of Tavolzhan Lake (North Kazakhstan). Mong. Acad. Sci. 2013, 10, 220-223. (In Russian)

64. Ongun Sevindik, T.; Tunca, H.; Gönülol, A.; Yildirim Gürsoy, N.; Küçükkaya, Ş.; Durgut Kinali, Z. Phytoplankton dynamics and structure, and ecological status estimation by the $Q$ assemblage index: A comparative analysis in two shallow Mediterranean lakes. Turk. J. Bot. 2017, 41, 25-36. [CrossRef]

65. Pasztaleniec, A.; Poniewozik, M. Phytoplankton based assessment of the ecological status of four shallow lakes (Eastern Poland) according to Water Framework Directive-A comparison of approaches. Limnologica 2010, 40, 251-259. [CrossRef]

66. Silvoso, J.; Izaguirrea, I.; Allende, L. Picoplankton structure in clear and turbid eutrophic shallow lakes: A seasonal study. Limnologica 2011, 41, 181-190. [CrossRef] 
67. Barinova, S.; Krupa, E.; Romanova, S. The role of planktonic algae in the ecological assessment of storage-reservoirs of the Ile-Balkhash basin (Kazakhstan). Transylv. Rev. Syst. Ecol. Res. Wetl. Divers. 2018, 20, 1-14.

68. Borics, G.; Nagy, L.; Miron, S.; Grigorszky, I.; László-Nagy, Z.; Lukács, B.; G-Tóth, L.; Várbíro, G. Which factors affect phytoplankton biomass in shallow eutrophic lakes? Hydrobiologia 2013, 714, 93-104. [CrossRef]

69. Van Ginkel, C.E.; Hohls, B.C.; Vermaak, E.A. Ceratium hirundinella (O.F. Muller) bloom in Hartbeespoort Dam, South Africa. Water SA 2001, 27, 269-276. [CrossRef]

70. Tomec, M.; Ternjej, I.; Kerovec, M.; Teskeredzic, E.; Meštrov, M. Plankton in the oligotrophic Lake Vrana (Croatia). Biol. Sect. Bot. 2002, 57, 579-588.

71. Grigorszky, I.; Padisák, J.; Borics, G.; Schitchen, C.; Borbély, G. Deep chlorophyll maximum by Ceratium hirundinella (O.F. Muller) Bergh in a shallow oxbow in Hungary. Hydrobiologia 2003, 506-509, 209-212. [CrossRef]

72. Jeppesen, E.; Jensen, J.P.; Sondergaard, M.; Lauridsen, T.; Landkildehus, F. Trophic structure, species richness and biodiversity in Danish lakes. Changes along a phosphorus gradient. Freshwater Biol. 2000, 45, 201-218. [CrossRef]

73. Stefanidis, K.; Parastergiadou, E. Influence of hydrophyte abundance on the spatial distribution of zooplankton in selected lakes in Greece. Hydrobiology 2010, 656, 55-65. [CrossRef]

74. Czerniawski, R.; Domagała, J. Reduction of zooplankton communities in small lake outlets in relation to abiotic and biotic factors. Ocean Hydro. 2013, 42, 123-131. [CrossRef]

75. Ferrara, O.; Vagaggini, B.; Margaritora, F. Zooplankton abundance and diversity in Lake Bracciano, Latium, Italy. J. Limnol. 2002, 61, 169-175. [CrossRef]

76. Degefu, F.; Schagerl, M. Zooplankton abundance, species composition and ecology of tropical high-mountain crater Lake Wonchi, Ethiopia. J. Limnol. 2015, 74, 324-334. [CrossRef]

77. John, B.R.; Arp, C.D.; Claudia, E.T.; Benjamin, M.J.; Matthew, S.W.; Thomas, R.R.; Erin, E.S.; David, M.O.; Kyle, C.S. Potential shifts in zooplankton community structure in response to changing ice regimes and hydrologic connectivity. Arct. Antarct. Alp. Res. 2019, 51, 327-345.

78. Adamczuk, M.; Kornijów, R. Crustacean communities as food resources for fish in shallow Polesie lakes with contrasting development of submerged macrophytes. Ocean Hydrol. 2011, 40, 11-18. [CrossRef]

79. Jeppesen, E.; Jensen, J.P.; Søndergaard, M.; Lauridsen, T.; Pedersen, L.J.; Jensen, L. Top-down control in freshwater lakes: The role of fish, submerged macrophytes and water depth. Hydrobiologia 1997, 343, 151-164. [CrossRef]

80. Williams, A.E.; Moss, B. Effect of different fish species and biomass on plankton interactions in a shallow lake. Hydrobiologia 2003, 491, 331-346. [CrossRef]

81. Alain, P.D.; Brenda, J.H. Warm spring and summer water temperatures in small eutrophic lakes of the Canadian prairies: Potential implications for phytoplankton and zooplankton. J. Plankton Res. 2009, 31, 489-502.

82. Hu, B.; Hu, X.; Nie, X.; Zhang, X.; Wu, N.; Hong, Y.; Qin, H. Seasonal and inter-annual community structure characteristics of zooplankton driven by water environment factors in a sub-lake of Lake Poyang, China. Peer] 2019, 7, e7590. [CrossRef] [PubMed]

83. Kuczynska-Kippen, N.; Joniak, T. Zooplankton diversity and macrophyte biometry in shallow water bodies of various trophic state. Hydrobiologia 2016, 774, 39-51. [CrossRef]

84. Ferrão-Filho, A.S.; Domingos, P.; Sandra, M.F.O.; Azevedo, O.; Domingo, P. Influences of a Microcystis aeruginosa Kotznig bloom on zooplankton populations in Jacarepaguli Lagoon (Rio de Janeiro, Brazil). Limnologica 2002, 32, 295-309. [CrossRef]

85. Rosińska, J.; Romanowicz-Brzozowska, W.; Kozak, A. Zooplankton changes during bottom-up and top-down control due to sustainable restoration in a shallow urban lake. Env. Sci. Pollut Res. 2019, 26, 19575-19587. [CrossRef] [PubMed]

86. Bockwoldt, K.A.; Nodine, E.R.; Mihuc, T.B.; Shambaugh, A.D.; Stockwell, J.D. Reduced Phytoplankton and Zooplankton Diversity Associated with Increased Cyanobacteria in Lake Champlain, USA. J. Contemp. Water Res. Educ. 2017, 160, 100-118. [CrossRef]

87. Horn, W. Investigations into the Food Selectivity of the Planktic Crustaceans Daphnia hyalina, Eudiaptomus gracilis and Cyclops vicinus. Hydrobiology 1985, 70, 603-612.

88. Zhang, M.; Smyth, R.A.; Zhu, W. Spatial distribution and filtering efficiency of Daphnia in a deep subtropical reservoir. J. Ocean. Limnol. 2019, 37, 1277-1288. [CrossRef]

89. Hopp, U.; Maier, G. Survival and development of five species of cyclopoid copepods in relation to food supply: Experiments with algal food in a flow-through system. Freshw. Biol. 2005, 50, 1454-1463.

90. Fileto, C.; Arcifa, M.S.; Henry, R.; Ferreira, A.R. Effects of temperature, sestonic algae features, and seston mineral content on cladocerans of a tropical lake. J. Limnol. 2010, 46, 135-147. [CrossRef]

91. Masclaux, H.; Bec, A.; Kainz, M.J.; Desvilettes, C.; Jouve, L.; Bourdier, G. Combined effects of food quality and temperature on somatic growth and reproduction of two freshwater cladocerans. Limnol. Oceanogr. 2009, 54, 1323-1332. [CrossRef]

92. Ernst, A.; Deicher, M.; Peter, M.; Herman, U. Nitrate and Phosphate Affect Cultivability of Cyanobacteria from Environments with Low Nutrient Levels. Wollenzien Appl. Environ. Microbiol. 2005, 71, 3379-3383. [CrossRef] [PubMed]

93. Ger, K.A.; Urrutia-Cordero, P.; Frost, P.C.; Hansson, L.A.; Sarnelle, O.; Wilson, A.E.; Lurling, M.; Hansson, L. The interaction between cyanobacteria and zooplankton in a more eutrophic world. Harm. Algae 2006, 54, 128-144. [CrossRef] [PubMed]

94. Landa, G.G.; Barbosa, F.A.; Rietzler, A.C.; Maia-Barbosa, P.M. Thermocyclops decipiens (Kiefer, 1929) (Copepoda, Cyclopoida) as Indicator of Water Quality in the State of Minas Gerais, Brazil. Braz. Arch. Biol. Technol. 2007, 50, 695-705. [CrossRef]

95. Rietzler, A.C.; Espíndola, E.L. Microcystis as a food source for copepods in a subtropical eutrophic reservoir. Verh. Intern. Ver. Limnol. 1998, 26, 2001-2005. [CrossRef] 
96. DeMott, W.R.; Gulati, R.D.; Donk, E.V. Daphnia food limitation in three hyperhypereutrophic Dutch lakes: Evidence for exclusion of large-bodied species by interfering filaments of cyanobacteria. Limnol. Oceanogr. 2001, 46, 2054-2060. [CrossRef]

97. Jang, M.H.; Ha, G.J.; Joo, N.T. Toxin production of cyanobacteria is increased by exposure to zooplankton. Freshwat. Biol. 2008, 48, 1540-1550. [CrossRef]

98. Frey, D.G. The penetration of cladocerans into saline waters. Hydrobiologia 1993, 267, 233-248. [CrossRef]

99. Bielańska-Grajner, I.; Cudak, A. Effects of Salinity on Species Diversity of Rotifers in Anthropogenic Water Bodies. Pol. J. Environ. Stud. 2014, 23, 27-34.

100. Ignoffo, T.R.; Bollens, S.M.; Bochdansky, A.B. The effects of thin layers on the vertical distribution of the rotifer, Brachionus plicatilis. J. Exp. Mar. Biol. Ecol. 2005, 316, 167-181. [CrossRef]

101. Alimov, A.F. Changes in the structure of animal communities accompanying eutrophication and pollution of aquatic ecosystems, Dokl. Biol. Sci. 2010, 433, 249-251.

102. Whittington, J.; Sherman, B.; Green, D. Growth of Ceratium hirundinella in a subtropical Australian reservoir: The role of vertical migration. J. Plankton Res. 2000, 22, 1025-1045. [CrossRef]

103. Sládecěk, V. Rotifers as indicators of water quality. Hydrobiologia 1983, 100, 169-201. [CrossRef]

104. Park, G.S.; Marshall, H.G. Estuarine relationships between zooplankton community structure and trophic gradients. J. Plankton Res. 2000, 22, 121-135. [CrossRef]

105. Turton, C.L.; McAndrews, J.H. Rotifers in second millennium sediment of Crawford Lake, Ontario, Canada. Rev. Palaeobot. Palynol. 2006, 141, 1-6. [CrossRef]

106. Zakaria, H.Y.; Hussien, A.M.; Flower, R. Environmental assessment of spatial distribution of zooplankton community in Lake Manzalah, Egypt. Acta Adriatica 2007, 48, 161-172.

107. Arndt, H. Dynamics and production of a natural population of Brachionus plicatilis (Rotatoria, Monogononta) in a eutrophicated inner coastal water of the Baltic. Kiel. Meeresforsch. Sonderh. 1988, 6, 147-153.

108. Xie, Z.; Xiao, H.; Tang, X.; Lu, K.; Cai, H. Interactions between red tide microalgae and herbivorous zooplankton: Effects of two bloom-forming species on the rotifer Brachionus plicatilis (O.F. Muller). Hydrobiologia 2008, 600, 237-245. [CrossRef]

109. Zou, Y.; Yamasaki, Y.; Matsuyama, Y.; Yamaguchi, K.; Honjo, T.; Oda, T. Possible involvement of hemolytic activity in the contact-dependent lethal effects of the dinoflagellate Karenia mikimotoi on the rotifer Brachionus plicatilis. Harm. Algae 2010, 9 , 367-373. [CrossRef]

110. Krupa, E.; Romanova, S.; Berkinbaev, G.; Yakovleva, N.; Sadvakasov, E. Zooplankton as Indicator of the Ecological State of Protected Aquatic Ecosystems (Lake Borovoe, Burabay National Nature Park, Northern Kazakhstan). Water 2020, $12,2580$. [CrossRef]

111. Kimambo, O.N.; Gumbo, J.R.; Chikoore, H. The occurrence of cyanobacteria blooms in freshwater ecosystems and their link with hydro-meteorological and environmental variations in Tanzania. Heliyon 2019, 5, 1-23. [CrossRef]

112. Sikora, A.; Dawidowicz, P. Breakage of cyanobacterial filaments by small- and large-sized Daphnia: Are there any temperaturedependent differences? Hydrobiologia 2017, 798, 119-126. [CrossRef] 